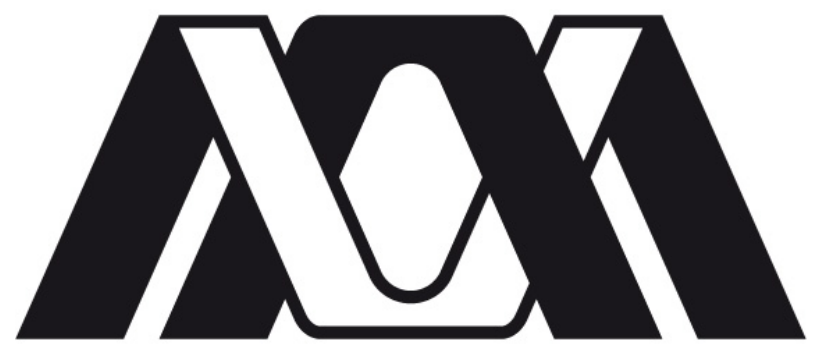

DIVISIÓN EN CIENCIAS BÁSICAS E INGENIERÍA Universidad Autónoma Metropolitana

Posgrado en Ciencias Matemáticas

UNIDAD IZTAPALAPA

\title{
Teoría de Juegos $S$-modulares con Aplicaciones a Sistemas de Espera en Tándem
}

Tesis que presenta:

JORGE DIEGO FUENTES MORA

Para obtener el grado de:

MAESTRO EN CIENCIAS MATEMÁTICAS

Asesor: DR. JOSÉ RAÚL MONTES DE OCA MACHORRO

Jurado calificador

Presidente: Doctor Julio Cesar García Corte

Secretario: Doctor José Raúl Montes de Oca Machorro

Vocal: Doctor Victor Hugo Vázquez Guevara

Ciudad de México, 11 de Noviembre de 2019 



\section{Agradecimientos}

A mi madre que durante toda mi vida siempre me ha brindado su apoyo y cariño, en especial durante esta etapa, a mis hermanos (Amdry, Marisol y Oswaldo) que siempre he recibido apoyo incondicional.

A mi novia Viridiana que siempre he recibido apoyo, motivación y amor incondicional.

A la Universidad Autónoma Metropolitana al abrirme sus puertas y haberme forjado como Matemático.

Al CONACYT por haberme apoyado económicamente durante mi estancia en este posgrado.

Al Doctor Raùl Montes de Oca por guiarme, orientarme y soportarme durante el tiempo que estudié la maestría y concluir con esta tesis

A la Doctora Patricia Saavedra por darme la oportunidad de estudiar este posgrado y motivarme a estudiar esta Maestrìa.

A los amigos que estaban, que hice y que siguen durante todo este tiempo.

¡A todos, muchas gracias! 



\section{Índice general}

$\begin{array}{ll}\text { Agradecimientos } & 3\end{array}$

Introducción $\quad 1$

1. Preliminares de Sistemas de Espera 4

1.1. Introducción . . . . . . . . . . . . . . . . . . . . . 4

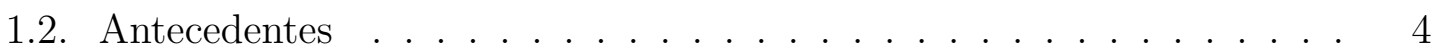

1.3. Sistemas de espera clásicos . . . . . . . . . . . . . . . . . . 7

1.3.1. Sistema de colas $M / M / 1$. . . . . . . . . . . . . . 7

1.3.2. Sistema de colas $M / M / S$. . . . . . . . . . . . . . . . . 9

1.4. Teorema de Burke . . . . . . . . . . . . . . . . . . . 11

1.5. Sistema de colas en tándem . . . . . . . . . . . . . . . . . . . 14

1.6. Conclusiones . . . . . . . . . . . . . . . . . . . . . . . . . . 18

2. Juegos Supermodulares 19

2.1. Introducción . . . . . . . . . . . . . . . . . . . . . . . . . 19

2.2. Látices . . . . . . . . . . . . . . . . . . . . . . . . . . . 19

2.2.1. Conjuntos parcialmente ordenados . . . . . . . . . . . . 19

2.2.2. Látices, sublátices y propiedades . . . . . . . . . . . . . . . . . 21

2.2.3. Propiedades topológicas de látices . . . . . . . . . . . . . . . . . 24

2.2.4. Orden inducido de un conjunto . . . . . . . . . . . . . 26

2.2.5. Puntos fijos . . . . . . . . . . . . . . . 28

2.3. Funciones supermodulares . . . . . . . . . . . . . . . . . . 29

2.3.1. Conjunto de soluciones óptimas . . . . . . . . . . . . . . . . . 32

2.4. Definición de juego supermodular . . . . . . . . . . . . . . . . 34

2.5. Existencia de puntos de equilibrio . . . . . . . . . . . . . . . . . . . 37

2.6. Algoritmo de optimización Round-Robin . . . . . . . . . . . . . . . . 39

2.7. Conclusiones . . . . . . . . . . . . . . . . . . . . . 40 
3. Juegos $S$-modulares y Ejemplos de Sistemas de Espera 41

3.1. Introducción . . . . . . . . . . . . . . . . . 41

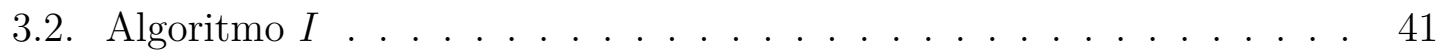

3.2.1. Monotonicidad, continuidad y estabilidad . . . . . . . . . 43

3.2.2. Estabilidad . . . . . . . . . . . . . . . . 43

3.2.3. Algoritmo $I$ para funciones $S$-modulares . . . . . . . . . . . . 44

3.2.4. Algoritmo para $n$ jugadores . . . . . . . . . . . . . . . . 49

3.3. S-modularidad . . . . . . . . . . . . . . 50

3.4. Optimizadores ordenados . . . . . . . . . . . . . . 52

3.5. Bifurcación . . . . . . . . . . . . . . . . 56

3.6. Conclusiones . . . . . . . . . . . . . . . . . . . . 58

4. Conclusiones y Perspectivas $\quad 59$

4.1. Conclusiones . . . . . . . . . . . . . . . . . . . . . . 59

4.2. Perspectivas . . . . . . . . . . . . . . . 60

$\begin{array}{lr}\text { Bibliografía } & 61\end{array}$ 


\section{Introducción}

En esta tesis se abordarán temas de Teoría de Juegos [15], a partir de lo cual se desarrolla una variante de ésta, la cual es la Teoría de Juegos S-modulares [21], en particular, estudiaremos el caso de juegos no-cooperativos. Usando esta teoría se desarrolla el modelado de Líneas de Espera en Tándem, comenzando con las nociones básicas de cada una de las teorías (Teoría de Juegos y Teoría de Colas) hasta abordar lo que son los juegos no cooperativos con estructura de $S$-modularidad, donde a partir de ciertas condiciones en el espacio de estrategias y las funciones de pago de cada uno de los jugadores, obtendremos la existencia, la estabilidad y la unicidad de los puntos de equilibrio de Nash en dichos juegos.

En los juegos $S$-modulares podemos encontrar una amplia variedad de aplicaciones:

a) en redes inalámbricas de comunicación [3] y [7],

b) en la programación del consumo de energía de un hogar [2],

c) el consumo de energía residencial [19],

d) en las redes tácticas radio cognitivas [16], etcétera.

Los objetivos de este trabajo son:

i) Analizar y desarrollar los juegos $S$-modulares a partir de la interpretación de los juegos supermodulares y submodulares, observando que a través de las propiedades de estos juegos obtenemos equilibrios de Nash.

ii) Estudiar los sistemas de líneas de espera a partir de la Teoría de Juegos $S$ modulares no-cooperativos, con la cual nos permitirá comenzar con tasas de servicio variables y no fijas, obteniendo de esta manera el control óptimo de 
estos Sistemas de Espera en Tándem, maximizando las funciones de pago de los servidores, interpretando condiciones que garantizarán existencia, unicidad y estabilidad de los puntos de equilibrio en este tipo de Sistemas Tándem, sin necesidad de hacer uso de los Teoremas de punto fijo (Kakutani [11], Brouwer $[24])$.

A partir de esta tesis se podrá conocer y analizar ciertas aplicaciones de la Teoría de Colas (algunos ejemplos podemos encontrarlos en [1], [8], [10], [12]) en particular en los Sistemas de Espera Tándem sin salida comenzando con el desarrollo de la Teoría de Juegos $S$-modulares [21] desde la interpretación de látices y sublátices, siguiendo con la caracterización de los juegos de equilibrio y hasta llegar al algoritmo para obtener los puntos de equilibrio de los juegos supermodulares, que nos servirán como referencia para el desarrollo de los juegos $S$-modulares, ya que la $S$ modularidad es un caso general de los juegos supermodulares y submodulares.

En consecuencia de lo anterior se desarrollará la teoría general de la Teoría de Colas en Tándem enfocándonos principalmente en los sistemas de Líneas de Espera en Tándem sin salidas entre los servidores, para después combinar las dos teorías y desarrollar un sistema de líneas de espera como un juego $S$-modular, donde los jugadores serán los servidores, de esta manera obtener tasas de servicio óptimas.

En el capítulo 1, desarrollaremos ciertos tipos de sistemas de espera, iniciando por el principal y básico sistema de líneas de espera $M / M / 1$, donde este sistema describe entradas y salidas de tipo Poisson con atención por parte de un solo servidor, al igual que este sistema se expone el sistema de líneas de espera $M / M / S$, que al igual que el sistema $M / M / 1$ tiene entradas y salidas de tipo Poisson pero con atención a clientes por parte de $s$ servidores. Por último, se analiza el Sistema de Líneas de Espera en Tándem donde se presentará anticipadamente el Teorema de Burke y usando la información obtenida a través del desarrollo de las colas $M / M / 1$ y $M / M / S$, obtendremos el número promedio de clientes en el sistema y la cantidad promedio de tiempo que los clientes pasan en el Sistema Tándem, el cual es un sistema que describe una línea de espera con $n$ servidores conectados uno tras otro, donde la única salida es cuando se concluye el servicio en el último servidor.

En el capítulo 2, mostraremos y desarrollaremos definiciones importantes sobre la supermodularidad y la submodularidad, empezando desde las definiciones de látices y sublátices entre las cuales se mostrará la definición de monotonicidad, después proseguiremos con propiedades topológicas tales como compacidad y así definir la completez para látices, además de definir puntos fijos para funciones y correspondencias. 
Luego se mostrarán funciones supermodulares, el conjunto de soluciones óptimas de tales funciones, en seguida de estos temas desarrollaremos las definiciones de lo que es un juego supermodular, así como la existencia de los puntos de equilibrio en estos juegos (para juegos no cooperativos) y concluiremos este capítulo con los algoritmos de optimización de Round Robin para las condiciones de convergencia en los juegos supermodulares.

En el capítulo 3, se mostrarán las condiciones generales necesarias que determinan la existencia, estabilidad y unicidad de los puntos de equilibrio en juegos $S$ modulares, donde mostraremos condiciones generales para juegos cóncavos $n$-personales. También se abordarán ciertos ejemplos de la Teoría $S$-modular, aplicando ésta específicamente en sistemas de líneas de espera con servidores en la modalidad de Sistemas Tándem, se comienza con la representación del algoritmo Round Robin para un sistema con dos servidores y más adelante el algoritmo de Round Robin para un sistema con $n$-servidores, donde se analizará la $S$-modularidad que es la generalización de la supermodularidad y submodularidad simultáneamente, concluyendo con un caso de bifurcación en este tipo de juego obteniendo una condición que garantiza que el punto de equilibrio es único.

Finalmente en el capítulo 4 se presentarán las conclusiones y perspectivas de esta tesis. 


\section{Capítulo 1}

\section{Preliminares de Sistemas de Espera}

\subsection{Introducción}

Este capítulo está basado en [6], [10], [11], [12], [15], [17], [24] que se usan como referencia para desarrollar éste, comenzaremos explicando la notación clásica de la Teoría de Colas, empezando con una breve explicación de la notación de Kendall-Lee, después se señala la fórmula de Little y se desarrolla el sistema clásico de la Teoría de Colas, abordando y desarrollando el sistema $M / M / 1$; más adelante el sistema $M / M / S$ y a partir de estos sistema junto con el Teorema de Burke, extendemos al Sistema deCcolas Tándem junto con las fórmulas para calcular el número promedio de clientes en el sistema y la cantidad promedio de tiempo que un cliente pasa en el sistema.

\subsection{Antecedentes}

Para la descripción de cualquier sistema de colas requerimos de la especificación de tres partes:

1. Los procesos de arribo.

2. El mecanismo de servicio, tal como el número de servidores y la distribución de tiempo de servicio.

3. La disciplina de la cola.

Notación 1.1. Kendall-Lee

La notación $A / B / S / K$ es usada para clasificar un sistema de colas, donde: 
- A especifica el tipo de procesos de arribo.

- $B$ denota la distribución del tiempo de servicio.

- $s$ denota el número de servidores en el sistema.

- $K$ denota la capacidad del sistema (haciendo referencia al máximo número de clientes que puede haber en el sistema).

En muchas ocasiones $K$ no se especifica, se llega a considerar que la capacidad del sistema es ilimitada.

Algunas cantidades básicas del sistema de colas son las siguientes:

L: El número promedio de clientes en el sistema.

$L_{q}$ : El número promedio de clientes esperando en la cola.

$L_{s}: \quad$ El número promedio de clientes en el servicio.

$W$ : La cantidad promedio de tiempo que un cliente pasa en el sistema.

$W_{q}$ : La cantidad promedio de tiempo que un cliente pasa esperando en el sistema.

$W_{s}$ : La cantidad promedio de tiempo que un cliente pasa en servicio.

Muchas relaciones útiles entre las anteriores y otras cantidades de interés pueden ser obtenidas usando la siguiente identidad de costo básica.

Nota 1.2. La tasa promedio de entrada al sistema es igual a $\lambda$ por la cantidad promedio que paga un cliente que ingresa, donde $\lambda$ está dada por

$$
\lambda=\lim _{t \rightarrow \infty} \frac{X(t)}{t} .
$$

Donde $\lambda$ es la tasa promedio de llegada de clientes que ingresan y $X(t)$ denota el número de clientes que llegan al tiempo $t, t \geq 0$.

La Fórmula de Little está dada de la siguiente manera

$$
L=\lambda W,
$$

de donde suponemos que cada cliente paga una cierta cantidad por unidad de tiempo mientras se encuentra en el sistema, una consecuencia de 1.1 (ver [9], página 52). 
Observación 1.3. Una variante de la ecuación 1.1, mientras el cliente se encuentra en la cola produce la siguiente ecuación:

$$
L_{q}=\lambda W_{q}
$$

y de igual manera, mientras el cliente se encuentra en el sistema, la ecuación 1.1 produce la siguiente ecuación:

$$
L_{s}=\lambda W_{s}
$$

Observación 1.4. Las ecuaciones 1.2, 1.3 y 1.4 son válidas para casi todos los sistemas de colas, independientemente del proceso de llegada, el número de servidores o la disciplina de la cola (ver [5], página 2).

Definición 1.5. Diremos que un sistema de colas se encuentra en estado $S_{n}$ si hay $n$ clientes en el sistema incluyendo si estos estós están siendo atendidos por algún servidor.

Definición 1.6. Si el sistema está en el estado $S_{n}$, sólo puede hacer transición al estado $S_{n-1}$ o al estado $S_{n+1}$, con $n \geqslant 1$, que significa que un cliente completa el servicio y deja el sistema o mientras el cliente actual está siendo atendido otro cliente arriba al sistema, de $S_{0}$ la única transición únicamente puede ser a $S_{1}$.

Si el sistema se encuentra en el estado $S_{n}$ en el tiempo $t$, la probabilidad de transición al estado $S_{n+1}$ en el intervalo de tiempo $(t, t+\Delta t)$ es $a_{n} \Delta t$. Nos referiremos a $a_{n}$ como el parámetro de llegada (llamado parámetro de nacimiento).

Si el sistema se encuentra en el estado $S_{n}$ en el tiempo $t$, la probabilidad de transición al estado $S_{n-1}$ en el intervalo de tiempo $(t, t+\Delta t)$ es $d_{n} \Delta t$. Nos referiremos a $d_{n}$ como el parámetro de partida (llamado parámetro de muerte).

Notación 1.7. La probabilidad de que un sistema de colas se encuentre en el estado $S_{n}$ en el tiempo $t, t \geq 0$ es,

$$
p_{n}(t)=P\{N(t)=n\}, \quad n=0,1, \ldots
$$

Donde $N(t)$ es el proceso de Markov que toma el valor $n$ cuando el sistema de colas está en el estado $S_{n}$ :

A continuación presentamos ecuaciones fundamentales recursivas para $N(t)$, 


$$
\begin{aligned}
& p_{n}^{\prime}(t)=-\left(a_{n}+d_{n}\right) p_{n}(t)+a_{n-1} p_{n-1}(t)+d_{n+1} p_{n+1}(t), \quad n \geqslant 1 \\
& p_{0}^{\prime}(t)=-\left(a_{0}+d_{0}\right) p_{0}(t)+d_{1} p_{1}(t)
\end{aligned}
$$

Donde asumiremos que en el estado estable (ver [18], página 390) se cumple que:

$$
\lim _{t \rightarrow \infty} p_{n}(t)=p_{n}
$$

Haciendo que $p_{n}^{\prime}(t)=0$ y $p_{0}^{\prime}(t)=0$ en 1.6, obtenemos las siguientes ecuaciones recursivas de estado estable (ver [18], página 390).

$$
\left(a_{n}+d_{n}\right) p_{n}(t)=a_{n-1} p_{n-1}(t)+d_{n+1} p_{n+1}(t), \quad n \geqslant 1,
$$

y para el caso cuando $d_{0}=0$ :

$$
a_{0} p_{0}(t)=d_{1} p_{1}(t)
$$

Las ecuaciones 1.8 y 1.9 se conocen como ecuaciones de equilibrio de estado estable.

Resolviendo las ecuaciones 1.8 y 1.9 en términos de $p_{0}$, obtenemos de manera recursiva

$$
\begin{aligned}
p_{1} & =\frac{a_{0}}{d_{1}} p_{0}, \\
p_{n} & =\frac{a_{0} a_{1} \ldots a_{n-1}}{d_{1} d_{2} \ldots d_{n}} p_{0},
\end{aligned}
$$

donde $p_{0}$ puede ser determinado por

$$
\sum_{n=0}^{\infty} p_{n}=\left(1+\frac{a_{0}}{d_{1}}+\frac{a_{0} a_{1}}{d_{1} d_{2}}+\ldots\right) p_{0}=1
$$

\subsection{Sistemas de espera clásicos}

\subsubsection{Sistema de colas $M / M / 1$}

En esta sección presentaremos el sistema de colas más simple en los sistemas de espera, que es, el sistema de colas $M / M / 1$, éste tiene un proceso de arribo de tipo Poisson con tasa $\lambda$ (= tasa promedio de llegada) y el tiempo de servicio en este sistema se distribuye exponencialmente con parámetro $\mu$ (= tasa promedio de servicio), ésto se ejemplifica abajo en el diagrama de la Figura 1. 


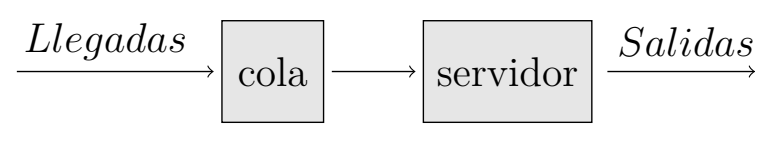

\section{Figura 1: Diagrama de un Sistema de Espera M/M/1.}

Entonces, este sistema de colas $M / M / 1$ en tiempo $t$ es un proceso de nacimiento y muerte con los siguientes parámetros de estado independientes:

$$
a_{n}=\lambda, \quad n \geqslant 0, \quad d_{n}=\mu, \quad n \geqslant 1 .
$$

Con estas restricciones y usando las ecuaciones $1.10,1.11$ y que $\lambda / \mu<1$ obtenemos

$$
\begin{gathered}
p_{0}=1-\frac{\lambda}{\mu}=1-\rho, \quad \text { y } \\
p_{n}=\left(1-\frac{\lambda}{\mu}\right)\left(\frac{\lambda}{\mu}\right)^{n}=(1-\rho) \rho^{n}, \quad n \geq 1 .
\end{gathered}
$$

Lo que implica que el servidor, en promedio, debe procesar a los clientes más rápido que el promedio de su tasa de llegada; de lo contrario, la longitud de la cola (el número de clientes que esperan en la cola) tiende a infinito. La relación $\rho=\lambda / \mu$ a veces se denomina intensidad de tráfico del sistema.

Definición 1.8. La intensidad de tráfico del sistema es:

Intensidad de tráfico $=\frac{\text { tiempo de servicio promedio }}{\text { tiempo de llegada promedio }}=\frac{\text { tasa de llegada promedio }}{\text { tasa de servicio promedio }}$.

Definición 1.9. El número promedio de clientes en el sistema $M / M / 1$ está dado por:

$$
L=\frac{\rho}{1-\rho}=\frac{\lambda}{\mu-\lambda} .
$$

Entonces, ajustando 1.2, 1.3 y 1.4, obtenemos, la cantidad promedio de tiempo que un cliente pasa en el sistema, la cantidad de tiempo promedio que un cliente pasa esperando en la cola y el número promedio de clientes esperando en la cola, 


$$
\begin{gathered}
W=\frac{1}{\mu-\lambda}=\frac{1}{\mu(1-\rho)}, \\
W_{q}=\frac{\lambda}{\mu(\mu-\lambda)}=\frac{\rho}{\mu(1-\rho)}, \\
L_{q}=\frac{\lambda^{2}}{\mu(\mu-\lambda)}=\frac{\rho^{2}}{(1-\rho)} .
\end{gathered}
$$

\subsubsection{Sistema de colas $M / M / S$}

En esta sección se desarrollará al igual que en la subsección anterior el sistema de colas $M / M / S$, que es un proceso de arribo de tipo Poisson con tasa $\lambda$ y cada uno de los $s$ servidores en el sistema tiene un tiempo de servicio exponencial con parámetro $\mu$, pero se trata con más servidores así como se muestra en el diagrama de la figura 2 , con 3 servidores.

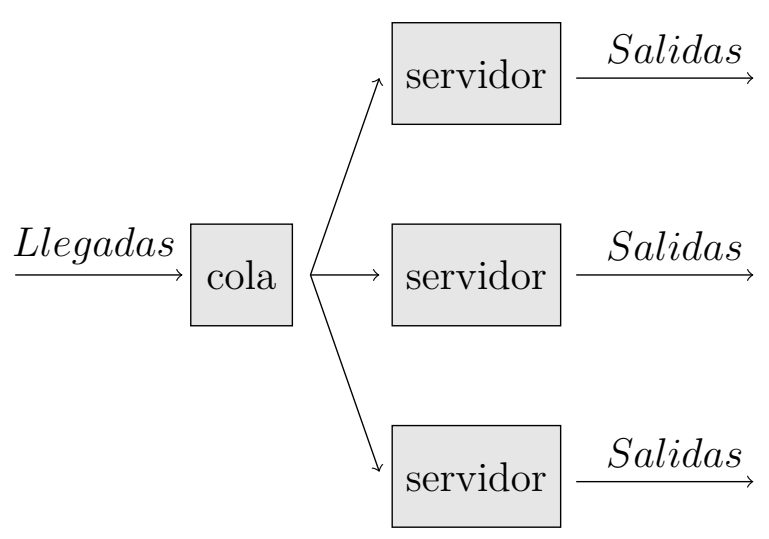

Figura 2: Diagrama de un sistema de espera $M / M / S$.

Entonces el sistema de colas $M / M / S$ en tiempo $t, t \geq 0$ es un proceso de nacimiento y muerte con los siguientes parámetros: 


$$
a_{n}=\lambda, \quad n \geqslant 0, \quad \begin{cases}n \mu, & 0<n<s \\ s \mu, & n \geqslant s .\end{cases}
$$

Tomar en cuenta que el parámetro de salida $d_{n}$ depende del estado del sistema. Entonces de las ecuaciones 1.10 y 1.11 obtenemos:

$$
\begin{gathered}
p_{0}=\left[\sum_{n=0}^{s-1} \frac{s \rho}{n !}+\frac{(s \rho)^{s}}{s !(1-\rho)}\right]^{-1}, \quad \mathrm{y} \\
p_{n}= \begin{cases}\frac{(s \rho)^{n}}{n !} p_{0}, & n<s \\
\frac{\rho^{n} s^{s}}{s !} p_{0}, & n \geqslant s,\end{cases}
\end{gathered}
$$

donde $\rho=\lambda /(s \mu)<1$.

Tomaremos en cuenta que la relación $\rho=\lambda /(s \mu)$ es la intensidad de tráfico del sistema de colas $M / M / S$. El número promedio de clientes en el sistema y el número promedio de clientes en la cola están dados, respectivamente, por:

$$
\begin{gathered}
L=\frac{\lambda}{\mu}+\frac{\rho(s \rho)^{s}}{s !(1-\rho)^{2}} p_{0}, \\
L_{q}=\frac{\rho(s \rho)^{s}}{s !(1-\rho)^{2}} p_{0}=L-\frac{\lambda}{\mu} .
\end{gathered}
$$

Por las ecuaciones 1.2 y 1.3 y las cantidades $W$ y $W_{q}$ están dados por: 


$$
\begin{gathered}
W=\frac{L}{\lambda}, \\
W_{q}=\frac{L_{q}}{\lambda}=W-\frac{1}{\lambda} .
\end{gathered}
$$

\subsection{Teorema de Burke}

En esta sección enunciaremos y demostraremos el Teorema de Burke, el cual será de gran importancia para desarrollar la sección posterior, que es el Sistema de Colas en Tándem. El Teorema de Burke demuestra que el proceso de salida de una cola $M / M / S$ sin salidas entre servidores, estable y estacionaria con tasa de llegada $\lambda$ es un proceso de Poisson de tasa $\lambda$ en el equilibrio.

Los detalles de la hipótesis son como sigue: suponemos una cola de una sola etapa con entrada aleatoria de tipo Poisson, el intervalo promedio entre llegadas tiene tamaño $1 / \lambda$, que es la probabilidad de que la llegada de un cliente durante un intervalo de tamaño $d t$ es tomado igual a $\lambda d t$, dentro de infinitesimales de orden superior, independiente del estado del sistema antes del tiempo t, tiempos de llegada de clientes anteriores o cualquier otra condición.

Bajo estas suposiciones y la condición adicional $s \mu / \lambda>1$, es bien sabido que hay una distribución de equilibrio de los estados (número de clientes en el sistema). Además esta distribución es la misma que la de los estados encontrados por los clientes que ingresan en el sistema.

Todos los clientes cuando entran al sistema permanecerán hasta que hayan recibido el servicio. De lo contrario, la disciplina de la cola o el orden del servicio es irrelevante, ya que la salida y no lo que ocasiona el retraso es de interés para el sistema de líneas de espera.

Con el fin de mostrar que la distribución de equilibrio del número de clientes completando el servicio durante un intervalo arbitrario de tamaño $T$ es Poisson con parámetro $\lambda T$, un resultado equivalente es que los intervalos de tiempo entre sucesivas finalizaciones de clientes son independientemente distribuidas con la misma distribución exponencial como los intervalos de tiempo entre llegadas, se obtendrá.

Debido a la aleatoriedad de la entrada y a la distribución exponencial de tiempo de retención, el proceso de salida es markoviano con respecto al estado del sistema, es 
decir, dado el estado del sistema en cualquier momento $t$, no hay más conocimiento sobre la distribución de salida subsecuente a $t$ se obtiene de la historia anterior del sistema. Se mostrará que un intervalo entre las partes y el estado del sistema al final del intervalo son independientes en el equilibrio. Junto con la propiedad markoviana, esta independencia implica que todos los intervalos entre las partes son independientes. Se mostrará simultáneamente que la distribución de equilibrio de un intervalo entre partidas es exponencial, y por lo tanto se sigue que la distribución de salida, o la distribución de terminaciones de los clientes, es Poisson.

Teorema 1.10. (Teorema de Burke)

Considere un sistema de colas con entrada de tipo Poisson y supongamos una línea de espera única sin abandonos con tiempos de servicio exponencial independientes distribuidos de manera idéntica. Entonces, la distribución de equilibrio del número de terminaciones de servicio en un intervalo de tiempo arbitrario será igual a la distribución de entrada (Poisson), para cualquier cantidad de servidores.

\section{DEMOSTRACIÓN}

Sea $T$ la variable aleatoria que representará el tiempo entre las salidas sucesivas (es decir, los tiempos entre partidas)

$$
F_{n}(t)=P\{N(t)=n, T>t\}
$$

entonce $F_{n}(t)$ es la probabilidad conjunta de que haya $n$ clientes en el sistema en un momento $t>0$ después de la última partida y que $t$ es menor que el tiempo entre partidas, es decir que no se ha producido otra partida. La distribución acumulada de la variable aletoria $T$, la cual denotaremos con $C(t)$, estará dada por:

$$
C(t)=P\{T \leq t\}=1-\sum_{n=0}^{\infty} F_{n}(t),
$$

el resultado anterior se da, considerando que: 


$$
\sum_{n=0}^{\infty} F_{n}(t)=P\{T>t\}
$$

Para encontrar $C(t)$, es necesario encontrar primero $F_{n}(t)$. Para esto, podemos escribir las siguientes ecuaciones de diferencia con respecto a $F_{n}(t)$ :

$$
\begin{aligned}
F_{n}(t+\Delta t)= & (1-\lambda \Delta t)(1-c \mu \Delta t) F_{n}(t)+\lambda \Delta t(1-c \mu \Delta t) F_{n-1}(t)+o(\Delta t), \\
& \text { cuando } c \leq n, \\
F_{n}(t+\Delta t)= & (1-\lambda \Delta t)(1-n \mu \Delta t) F_{n}(t)+\lambda \Delta t(1-n \mu \Delta t) F_{n-1}(t)+o(\Delta t), \\
& \text { cuando } 1 \leq n \leq c, y \\
F_{0}(t+\Delta t)= & (1-\lambda \Delta t) F_{0}(t)+o(\Delta t) .
\end{aligned}
$$

Restando $F_{n}(t)$ de cada lado de las ecuaciones anteriores y dividiendo ambos lados por $\Delta t$, y tomando el límite $\Delta t \rightarrow 0$, obtenemos las siguientes ecuaciones:

$$
\begin{aligned}
& \frac{d F_{n}(t)}{d t}=-(\lambda+c \mu) F_{n}(t)+\lambda F_{n-1}(t) \text { cuando } c \leq n, \\
& \frac{d F_{n}(t)}{d t}=-(\lambda+n \mu) F_{n}(t)+\lambda F_{n-1}(t) \text { cuando } 1 \leq n \leq c, \\
& \frac{d F_{0}(t)}{d t}=-(\lambda) F_{0}(t) .
\end{aligned}
$$

Usando la condición de límite

$$
\begin{gathered}
F_{n}(0)=P\{N(0)=n, T>0\}=P\{N(0)=n\}=p_{n}, \\
F_{n}(t)=p_{n} e^{-\lambda t},
\end{gathered}
$$


recordando que para modelos $M / M / S$ de la subsección 1.3.2, tenemos

$$
p_{n+1}= \begin{cases}\frac{\lambda}{(n+1) \mu} p_{n}, & 1 \leq n \leq c \\ \frac{\lambda}{c \mu} p_{n}, & c \leq n\end{cases}
$$

y de aquí que para obtener $C(t)$, que es la distribución acumulada del tiempo entre partidas usamos 1.28 y tenemos que:

$$
C(t)=1-\sum_{n=0}^{\infty} p_{n} e^{-\lambda t}=1-e^{-\lambda t} \sum_{n=0}^{\infty} p_{n}=1-e^{-\lambda t}
$$

de esta manera, mostramos que el tiempo entre partidas es exponencial.

Es también verdad que las variables aleatorias $N(T)$ y $T$ son independientes y que además que los tiempos de salida sucesivas entre partidas, uno del otro, son independientes.

Observación 1.11. El Teorema de Burke tiene aplicaciones en problemas de colas en Tándem, en capítulos posteriores se mostrarán algunos ejemplos.

\subsection{Sistema de colas en tándem}

Definición 1.12. Consideremos un sistema de 2 servidores en el cual los clientes llegan con una tasa $\lambda$ de tipo Poisson al servidor 1. Después de ser atendidos por el servidor 1, se unen a la cola frente al servidor 2. Suponiendo que el espacio de espera entre ambos servidores es infinito. Cada servidor sirve a un cliente a la vez con el servidor $i$ tomando un tiempo exponencial con tasa $\mu_{i}$ para un servicio, $i=1,2$. Tal sistema se llama tándem o sistema secuencial, (se ejemplifica este proceso a través del diagrama que se muestra en la figura 3).

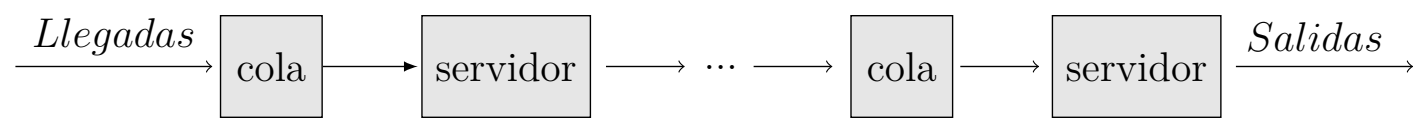




\section{Figura 3: Diagrama de un Sistema de Espera en Tándem.}

Para analizar este sistema, necesitamos hacer un seguimiento del número de clientes en el servidor 1 y en el servidor 2 .

Definición 1.13. Donde empezaremos por definir la probabilidad de transición en un sólo paso y la denotaremos como $P_{n, m}$ que será pasar del estado $n$ al estado $m$ en un paso.

Donde $(n, m)$ será la pareja que denota que hay $n$ clientes en el servidor 1 y $m$ en el servidor 2 y las ecuaciones de balance son las siguientes:

$$
\begin{array}{cc}
\begin{array}{c}
\text { Estado } \\
0,0
\end{array} & \text { Tasa en la cual el proceso sale }=\text { tasa en la que el proceso entra } \\
\lambda P_{0,0}=\mu_{2} P_{0,1} & \\
n, 0 ; n>0 & \left(\lambda+\mu_{1}\right) P_{n, 0}=\mu_{2} P_{n, 1}+\lambda_{2} P_{n-1,0} \\
0, m ; m>0 & \left(\lambda+\mu_{2}\right) P_{0, m}=\mu_{2} P_{0, m+1}+\mu_{1} P_{1, m-1} \\
n, m ; n m>0 & \left(\lambda+\mu_{1}+\mu_{2}\right) P_{n, m}=\mu_{2} P_{n, m+1}+\mu_{1} P_{n+1, m-1}+\lambda P_{n-1, m}
\end{array}
$$

En lugar de resolver estas ecuaciones directamente notamos que la situación en el servidor 1 es igual que en un modelo $M / M / 1$. Ya que si lo analizamos con el Teorema de Burke (enunciado y demostrado en la sección anterior), el cual nos menciona que, una cadena de nacimiento y muerte puede analizarse como una cadena de tiempo reversible y la salida de la cola $\mathrm{M} / \mathrm{M} / 1$ será un proceso de Poisson con tasa $\lambda$ y el servidor 2 tendrá un análisis parecido a una cola $M / M / 1$. Por lo tanto, la probabilidad de que haya $n$ clientes en el servidor 1 es:

$$
P(\text { n clientes en el servidor } 1)=\left(1-\frac{\lambda}{\mu_{1}}\right)\left(\frac{\lambda}{\mu_{1}}\right)^{n} \text {, }
$$


y similarmente

$$
P(\mathrm{~m} \text { clientes en el servidor } 2)=\left(1-\frac{\lambda}{\mu_{2}}\right)\left(\frac{\lambda}{\mu_{2}}\right)^{m} \text {. }
$$

Ahora, si el número de clientes en el servidor 1 y 2 son variables aleatorias independientes, entonces se sigue que:

$$
P_{n, m}=\left(\frac{\lambda}{\mu_{1}}\right)^{n}\left(1-\frac{\lambda}{\mu_{1}}\right)\left(\frac{\lambda}{\mu_{2}}\right)^{m}\left(1-\frac{\lambda}{\mu_{2}}\right) .
$$

Para verificar que $P_{n, m}$ es de hecho igual a lo anterior (y se cumple que por lo tanto el número de clientes en el servidor 1 es independiente del número de clientes en el servidor 2), todo lo que necesitamos hacer es verificar que lo anterior satisfaga las ecuaciones 1.30 esto es suficiente ya que sabemos que $P_{n, m}$ es la única solución de las ecuaciones 1.30.

Ahora L, el número promedio de clientes en el sistema, está dado por

$$
\begin{aligned}
L & =\sum_{n, m}(n+m) P_{n, m} \\
& =\sum_{n} n\left(1-\frac{\lambda}{\mu_{1}}\right)\left(\frac{\lambda}{\mu_{1}}\right)^{n}+\sum_{m} m\left(1-\frac{\lambda}{\mu_{2}}\right)\left(\frac{\lambda}{\mu_{2}}\right)^{m} \\
& =\frac{\lambda}{\mu_{1}-\lambda}+\frac{\lambda}{\mu_{2}-\lambda} .
\end{aligned}
$$

Y de aquí se podrá observar que el tiempo promedio que un cliente espera en el sistema es:

$$
W=\frac{L}{\lambda}=\frac{1}{\mu_{1}-\lambda}+\frac{1}{\mu_{2}-\lambda} .
$$


Observación 1.14. El resultado 1.30 pudo haber sido obtenido como consecuencia de la reversibilidad (ver [18], página 250) del sistema $M / M / 1$. Porque la reversibilidad del tiempo no sólo implica que la salida del servidor 1 es un proceso de Poisson, sino que también implica que el número de clientes en el servidor 1 es independiente de los tiempos de salida anteriores del servidor 1 y estos últimos tiempos de salida constituyen el proceso de llegada al servidor 2 .

El resultado anterior puede ser sustancialmente generalizado. Para hacerlo, consideremos un sistema de $k$ servidores. Donde los clientes llegan desde el exterior del sistema al servidor $i, i=1, \ldots, k$, con una tasa $r_{i}$; luego se unen a la cola en $i$ hasta que llega su turno al servicio. Una vez que el servidor $i$ atiende a un cliente, éste se une a la cola frente al servidor $j, j=1, \ldots, k$, con probabilidad $P_{i j}$. Por tanto, si $\sum_{j=1}^{k} P_{i j} \leq 1$ representa la suma de todas las probabilidades de pasar del servidor $i$ a los demás servidores, entonces, $1-\sum_{j=1}^{k} P_{i j}$ representará la probabilidad de que un cliente abandone el sistema después de ser atendido por el servidor $i$ :

$$
\lambda_{j}=r_{j}+\sum_{i=1}^{k} \lambda_{i} P_{i j}, \quad i=1, \ldots, k
$$

La ecuación anterior se sigue de que $r_{j}$ es la tasa de llegada de los clientes a $j$ viniendo del exterior del sistema y como $\lambda_{i}$ es la tasa en la que salen los clientes del servidor $i$ (igual a la tasa de salida), $\lambda_{i} P_{i j}$ será la tasa de llegada al servidor $j$ viniendo desde el servidor $i$. Resulta que el número de clientes en cada uno de los servidores es independiente y de la forma:

$$
P\{\text { n clientes en el servidor } \mathrm{j}\}=\left(1-\frac{\lambda_{j}}{\mu_{j}}\right)\left(\frac{\lambda j}{\mu j}\right)^{n},
$$

donde $\mu_{j}$ es la tasa de servicio exponencial en el servidor $j$ y las $\lambda_{j}$ son la solución para la ecuación 1.33. Por supuesto, es necesario que $\frac{\lambda_{j}}{\mu_{j}}<1, \forall j$. Para probar esto, primero notamos que es equivalente afirmar que las probabilidades limitantes $P\left(n_{1}, n_{2}, \ldots, n_{k}\right)=P\left\{n_{j}\right.$ en el servidor $\left.j, j=1, \ldots, k\right\}$, están dadas por: 


$$
P\left(n_{1}, n_{2}, \ldots, n_{k}\right)=\prod_{j=1}^{k}\left(\frac{\lambda j}{\mu j}\right)^{n}\left(1-\frac{\lambda_{j}}{\mu_{j}}\right)
$$

la cual puede ser verificada mostrando que satisface las ecuaciones de balance para este modelo. El número promedio de clientes en el sistema es:

$$
\begin{aligned}
L & =\sum_{i=1}^{k} \text { número promedio en el servidor } j \\
& =\sum_{i=1}^{k} \frac{\lambda_{j}}{\mu_{j}-\lambda_{j}} .
\end{aligned}
$$

El tiempo promedio que un cliente espera en el sistema puede ser obtenido de $L=\lambda W \operatorname{con} \lambda=\sum_{j=1}^{k} r_{j}$ y esto da paso a que $W$ pueda representarse como:

$$
W=\frac{\sum_{i=1}^{k} \frac{\lambda_{j}}{\mu_{j}-\lambda_{j}}}{\sum_{j=1}^{k} r_{j}} .
$$

Observación 1.15. El resultado incorporado en la ecuación 1.34 nos muestra que la distribución del número de clientes en el servidor $i$ es la misma que en un sistema $M / M / 1$ con tasas $\lambda_{i}$ y $\mu_{i}$

\subsection{Conclusiones}

En este capítulo desarrollamos los sistemas de espera $M / M / 1$ en equilibrio y el Teorema de Burke, que nos dice que la distribución del tiempo entre salidas consecutivas de este sistema es idéntica a la distribución del tiempo entre llegadas. De esta manera obtenemos que la distribución de las salidas es la misma que la distribución de las llegadas y no se afecta por el mecanismo de servicio (exponencial) y entonces el análisis de un Sistema de Espera en Tándem es completamente parecido al sistema $M / M / 1$. 


\section{Capítulo 2}

\section{Juegos Supermodulares}

\subsection{Introducción}

Esta sección está basada en: [2], [13], [17], [20], [21], [22] los cuales se tomaron como referencia para la explicación y el desarrollo de la supermodularidad, hasta obtener un algoritmo que nos permita obtener un criterio de existencia de equilibrios, empezando con la construcción de la Teoría de los Juegos Supermodulares, definiendo los látices, propiedades de puntos fijos, funciones supermodulares que se ocuparán de base para funciones $S$-modulares, entre otras hasta llegar al algoritmo de Round Robin, el cual nos dice que por medio de ciertas condiciones en el espacio de estrategias de cada uno de los jugadores, llegamos a la existencia de puntos de equilibrio que nos garantiza un equilibrio de Nash sin ocupar directamente los Teoremas de punto de fijo de Brouwer ([24], página 222) o de Kakutani [11].

\subsection{Látices}

\subsubsection{Conjuntos parcialmente ordenados}

En esta subsección comenzaremos definiendo algunas condiciones de interés y útiles como son: relación binaria, conjunto parcialmente ordenado y cotas para conjuntos, para así poder definir el concepto de látiz. Primero, daremos a conocer el concepto de relación binaria, para que más adelante definamos lo que es un conjunto parcialmente ordenado y se darán algunos ejemplos de las definiciones dadas.

Definición 2.1. Diremos que $\preceq$ es una relación binaria sobre un conjunto $X$, si para cualesquiera $x, y \in X$, se tiene que $x \preceq y$ es verdadero ó $x \preceq y$ es falso. 
Definición 2.2. $X$ es un conjunto parcialmente ordenado si en él hay una relación binaria $\preceq$ que cumple las siguientes propiedades:

a) Reflexividad: si $x \preceq x$ para cada $x \in X$.

b) Transitividad: si $x \preceq y$ y $y \preceq z$ implican que $x \preceq z$, para $x, y, z \in X$.

c) Antisimetría: si $x \preceq y$ y $y \preceq x$ implican que $x=y$, para $x, y \in X$.

A $\preceq$ se le llama relación de orden.

Ejemplo 2.3. Los siguientes son conjuntos parcialmente ordenados:

1. $\mathbb{R}$ con la relación $\leq$, es decir, el orden usual en $\mathbb{R}$.

2. Dado un conjunto $X$, el conjunto potencia, denotado por $\mathscr{P}(X)$, con la relación $\subseteq$.

3. Sea $I$ un conjunto de índices y $X_{i}$ un conjunto parcialmente ordenado con la relación $\preceq_{i}$, para cada $i \in I$; entonces el ejemplo es, el producto directo $\prod_{i \in I} X_{i}$, con la relación $\preceq$, dada por $x \preceq y$, para $x, y \in \prod_{i \in I} X_{i}$, si $x_{i} \preceq_{i} y_{i}$, para cada $i \in I$.

Ya que en un conjunto parcialmente ordenado, no siempre se cumple que $x \preceq y$ ó $y \preceq x$, para cualquier par $x, y$ en él, esto da lugar a lo siguiente:

Definición 2.4. Se dice que $x, y$ son comparables en $X$ si $x \preceq y$ ó $y \preceq x$, en otro caso, $x$ y $y$ son incomparables.

Definición 2.5. Un conjunto parcialmente ordenado es una cadena si todos sus elementos son comparables.

A continuación mencionaremos algunas nociones de cotas en conjuntos parcialmente ordenados, para tener un acercamiento al concepto de látiz.

Definición 2.6. Dado un conjunto parcialmente ordenado $X$ y sea $X^{\prime}$ un subconjunto dado de $X$. Diremos que $y \in X$ es una cota superior (inferior) de $X^{\prime}$ si $x \preceq y(y \preceq x)$, para cada $x \in X^{\prime}$, si además, de lo anterior, se cumple que $y \in X^{\prime}$, entonces $y$ es el elemento máximo (mínimo) de $X^{\prime}$.

Definición 2.7. Se dice que $x \in X^{\prime}$ es un elemento maximal (minimal) de $X^{\prime}$ si no existe $y \in X^{\prime}$ tal que $x \preceq y(y \preceq x)$. 
Observación 2.8. Todo elemento máximo es maximal, de manera análoga, todo elemento mínimo es minimal.

Nota 2.9. En un conjunto parcialmente ordenado, los elementos máximos (mínimos), si existen, son únicos. En cambio, puede que en el conjunto exista más de un elemento maximal (minimal). Los elementos maximales (minimales) distintos no son comparables.

Definición 2.10. Si el conjunto de cotas superiores de $X$ tiene un elemento mínimo, esta mínima cota superior es llamada supremo de $X$; similarmente, la máxima cota inferior de $X$ es llamada infimo.

Notación 2.11. Si consideramos $X$ un conjunto parcialmente ordenado, y $x, y \in X$, y estos tienen una mínima cota superior en $X$, ésta se denota por $x \vee y$; de manera similar, la máxima cota inferior en $X$ de tales elementos, se denota por $x \wedge y$.

\subsubsection{Látices, sublátices y propiedades}

Para esta subsección, definiremos los conceptos de látiz y sublátiz que serán unos de los conceptos importantes para esta teoría. Usando las definiciones de la subsección 2.2.1 y estos conceptos en capítulos posteriores caracterizaremos los conjuntos de perfiles de estrategias admisibles en los juegos $S$-modulares.

Definición 2.12. Un conjunto parcialmente ordenado que contiene la máxima cota inferior $(\vee)$ y la mínima cota superior $(\wedge)$ de cualquier pareja de elementos de este conjunto, será denominado látiz.

Ejemplo 2.13. Los siguientes conjuntos son ejemplos de látices

1. $\mathscr{P}(X)$, de un conjunto $X$, con la relación binaria $\subseteq$. Es una látiz, pues, para $A, B$ subconjuntos de $X, A \vee B=A \cup B$ y $A \wedge B=A \cap B$.

2. $\mathbb{R}$, con la relación de orden $\leq$, donde, $x \vee y=\max \{x, y\}$ y $x \wedge y=\min \{x, y\}$, para $x, y \in \mathbb{R}$.

3. $\mathbb{R}^{n}$ con la relación $\leq$, donde, para $x, y \in \mathbb{R}^{n}, x \vee y=\left(x_{1} \vee y_{1}, \ldots, x_{n} \vee y_{n}\right)$ y $x \wedge y=\left(x_{1} \wedge y_{1}, \ldots, x_{n} \wedge y_{n}\right)$. Con $x_{i} \vee y_{i}$ y $x_{i} \wedge y_{i}$ definidas en el inciso 2 .

Observación 2.14. El producto directo de látices es una látiz. (ver [21], página 13) 
Definición 2.15. Si $Y$ es un subconjunto de una látiz $X$ y se cumple que para cualquier par de elementos contenidos en este subconjunto, $Y$ contiene su máxima cota inferior y su mínima cota superior, respecto al conjunto $X$, entonces diremos que $Y$ es una sublátiz de $X$.

A continuación, se muestran ejemplos y observaciones sobre sublátices.

Nota 2.16. Denotaremos con $\mathscr{L}(X)$ al conjunto de sublátices no vacías de una látiz $X$

Ejemplo 2.17. Los siguientes son sublátices

1. Cualquier subconjunto de $\mathbb{R}$.

2. Cualquier subconjunto de una cadena es una sublátiz de ésta.

Observación 2.18. Sobre sublátices.

a) Dada una látiz $X$ y $X^{\prime}$ una sublátiz de ésta, si $Y$ es una sublátiz de $X^{\prime}$, entonces también lo es de $X$.

b) Si $X$ y $Y$ son látices con la misma relación de orden y tales que $X \subset Y$, no siempre ocurre que $X$ sea una sublátiz de $Y$.

Para ejemplificar la segunda parte de la Observación 2.18, considere el siguiente ejemplo:

Ejemplo 2.19. Sea $X$ un conjunto no vacío y $Y=\mathscr{P}(X)$, se sabe que $Y$ es una látiz con la relación binaria $\subseteq$. Considere $Z=\{\emptyset, X, A, B\}$, con $A, B \subset X, A \neq B$ y $A \neq B^{c}$. Note que $Z \subset Y$, es una látiz con la relación $\subseteq$ pero, en $Z$ se tiene que $A \vee_{Z} B=X$ y $A \wedge_{Z} B=\emptyset$, en cambio $A \vee_{Y} B=A \cup B$ y $A \wedge_{Y} B=A \cap B$, de esto que $Z$ no sea una sublátiz de $Y$.

Definición 2.20. Una función $f$ definida de un conjunto parcialmente ordenado $X$ a otro conjunto parcialmente ordenado $Y$ es creciente (decreciente) si $x^{\prime} \preceq x^{\prime \prime}$ en $X$ implica que $f\left(x^{\prime}\right) \preceq f\left(x^{\prime \prime}\right)\left(f\left(x^{\prime}\right) \succeq f\left(x^{\prime \prime}\right)\right)$.

Diremos que una función es monótona si es una función, ya sea, creciente o decreciente 
Los siguientes lemas son algunas propiedades básicas de látices.

Lema 2.21. Si $Y$ es una sublátiz de una látiz $X$ y $Z$ un subconjunto de $Y$, finito y no vacío, entonces $\inf _{X} Z$ y $\sup _{X} Z$ existen y son elementos de $Y$. De esto que, si $X \neq \emptyset$ es una látiz finita, entonces $X$ tiene supremo e ínfimo. (la demostración puede verse en [21] página 16)

Lema 2.22. Si $X$ es una látiz y $X_{\alpha}$ es una sublátiz de $X$ para cada $\alpha \in A$, entonces $\bigcap_{\alpha \in A} X_{\alpha}$ es una sublátiz de $\mathrm{X}$.

\section{Demostración}

Supongamos $x, y \in \bigcap_{\alpha \in A} X_{\alpha}$, por hipótesis se cumple que $x, y \in X_{\alpha}$, para todo $\alpha \in A$, y cada $X_{\alpha}$ es sublátiz de $X$, de aquí que $x \vee y, x \wedge y \in X_{\alpha}$, para todo $\alpha \in A$, así $x \vee y, x \wedge y \in \bigcap_{\alpha \in A} X_{\alpha}$.

De esto se concluye que $\bigcap_{\alpha \in A} X_{\alpha}$ es una sublátiz de $X$.

Definición 2.23. Sean $X$ y $T$ látices, $S \subset X \times T$, la sección de $S$ sobre $t$ en $\mathrm{T}$, es el conjunto $S_{t}=\{x \in X:(x, t) \in S\}$, y la proyección de $S$ sobre $T$ es $\Pi_{T} S=\{t \in T$ : $\left.S_{t} \neq \emptyset\right\}$.

En seguida, se mostrarán un par de resultados sobre conjuntos en la Definición 2.23 .

Lema 2.24. Sean $X$ y $T$ látices, y $S$ una sublátiz de $X \times T$.

a) La sección $S_{t}$ de $S$ en cada $t \in T$ es una sublátiz de $X$.

b) La proyección $\Pi_{T} S$ de $S$ sobre $T$ es una sublátiz de $T$.

\section{DEMOSTRACIÓN}


a) Sea $t \in T$ y $x, y \in S_{t}$, luego $(x, t),(y, t) \in S$. Como $S$ es una sublátiz, $(x, t) \vee$ $(y, t) \in S$ y $(x, t) \wedge(y, t) \in S$, así $x \vee y, x \wedge y \in S_{t}$. De esto que $S_{t}$ sea una sublátiz de $X$.

b) Considere $t, u \in \Pi_{T} S$, se tiene que $S_{t}$ y $S_{u}$ son no vacíos; sean $s$ y $v$ en $S_{t}$ y $S_{u}$, respectivamente. $S$ es una sublátiz de $X \times T$, así que

$$
(s, t) \vee(v, u)=(s \vee v, t \vee u) \in S \quad \mathrm{y} \quad(s, t) \wedge(v, u)=(s \wedge v, t \wedge u) \in S
$$

Se sigue que, $S_{t \vee u}$ y $S_{t \wedge u}$ son no vacíos, luego $t \vee u$ y $t \wedge u$ están en $\Pi_{T} S$. Con esto se concluye que $\Pi_{T} S$ es una sublátiz de $T$.

\subsubsection{Propiedades topológicas de látices}

Para esta parte trataremos condiciones de compacidad, definiremos el concepto de completez para látices y daremos una caracterización de látices completas usando conceptos topológicos. Estos resultados serán de utilidad para asegurar la existencia de máximos y mínimos en los conjuntos de perfiles de mejor respuesta, para juegos supermodulares cuyos perfiles de estrategias admisibles son subconjuntos de $\mathbb{R}^{n}$.

Comenzaremos definiendo la completez para látices, después daremos algunas observaciones de látices completas y unos ejemplos.

Definición 2.25. Diremos que una látiz es completa, si cada subconjunto no vacío de esta látiz tiene supremo e ínfimo.

Nota 2.26. De la definición anterior y el Lema 2.21, tenemos que:

a) Cualquier látiz finita es completa.

b) Una látiz completa, no vacía, tiene supremo e ínfimo.

Ejemplo 2.27. Las siguientes son látices completas.

1. Los intervalos cerrados en $\mathbb{R}$.

2. $\mathscr{P}(X)$, para $X \neq \emptyset$. 
Haciendo uso del concepto de sublátiz y del concepto de completez, obtenemos la siguiente definición.

Definición 2.28. Sea $Y$ una sublátiz de una látiz $X$, diremos que $Y$ es una sublátiz

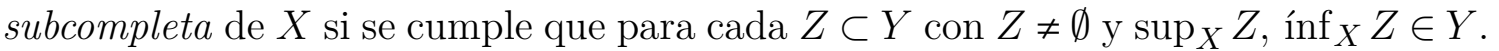

Ejemplo 2.29. Tomando las látices completas del ejemplo 2.27, se presentan las siguientes sublátices subcompletas de éstas.

1. Para los intervalos cerrados en $\mathbb{R}$, los conjuntos de la forma $[a, b] \cap \mathbb{N}$.

2. Para $\mathscr{P}(X)$, la colección $\left\{A, A^{C}, \emptyset, X\right\}$, con $A \subset X$.

Observación 2.30. Sobre látices completas.

a) Si $X$ es una látiz y $Y$ una sublátiz subcompleta de ésta, entonces $\sup _{X} Z=$ $\sup _{Y} Z$ e $\inf _{X} Z=\inf _{Y} Z$, para cualquier $Z \subset Y, Z \neq \emptyset$. Además, $Y$ es una látiz completa.

b) Si $X$ y $Y$ son látices completas con la misma relación de orden y $Y \subset X$, entonces $Y$ no necesariamente es una sublátiz subcompleta de $X$.

c) La intersección de una látiz completa (sublátiz subcompleta) y un intervalo cerrado, es una látiz completa (sublátiz subcompleta).

Para mostrar $b$ ) de la observación anterior, se tiene el siguiente.

Ejemplo 2.31. Recordando el ejemplo 2.19, para $X \neq \emptyset, \mathscr{P}(X)$ es una látiz completa. Si $Z=\{A, B, X, \emptyset\}$, con $A \neq B, A \neq B^{c}$ y $A, B \subset X$. Note que $Z \subset \mathscr{P}(X)$ y $Z$ es una látiz completa.

Considere $Y=\{A, B\}, Y \subset Z$, observe que $\sup _{Z} Y=X \neq \sup _{\mathscr{P}(X)} Y=A \cup B$. De esto se sigue que $Z$ no es una sublátiz subcompleta de $\mathscr{P}(X)$.

El siguiente teorema, muestra que subcompletez es equivalente a compacidad en la topología usual de $\mathbb{R}^{n}$.

Teorema 2.32. Una sublátiz de $\mathbb{R}^{n}$ es subcompleta si y sólo si es compacta, (la demostración puede verse en [21] página 30).

A partir del teorema recien enunciado, se puede deducir el siguiente resultado. 
Corolario 2.33. Una sublátiz de $\mathbb{R}^{n}$, compacta y no vacía, tiene un elemento máximo y un elemento mínimo.

El lema que se enuncia a continuación, provee un resultado para las sublátices no vacías y cerradas que son acotadas, ya sea superior o inferiormente.

Lema 2.34. Una sublátiz cerrada de $\mathbb{R}^{n}$ que es acotada superiormente (inferiormente), tiene un elemento máximo (mínimo).

La demostración del Lema 2.34, se puede encontrar en [21] (página 31, Corolario $2.3 .1)$.

\subsubsection{Orden inducido de un conjunto}

Para esta parte comenzaremos definiendo el orden inducido de un conjunto y enunciaremos algunos atributos de tal conjunto.

Definición 2.35. Sea $X$ una látiz con relación de orden $\preceq$. El orden inducido del conjunto $\sqsubseteq$, se define sobre la colección de elementos no vacíos del conjunto potencia de $X$, y es tal que para $A, B \in \mathscr{P}(X) \backslash\{\emptyset\}, A \sqsubseteq B$, si $x \in A$ y $y \in B$ implican que, $x \wedge y \in A$ y $x \vee y \in B$.

Los singuletes (es decir, conjuntos con un solo elemento) en $X$ son ordenados por $\sqsubseteq$ si y sólo si, los elementos son ordenados por $\preceq$; es decir, para $a, b \in X,\{a\} \sqsubseteq\{b\}$ si y sólo si $a \preceq b$.

Lema 2.36. Sean $X$ una látiz y $Y, Z$ subconjuntos no vacíos de $X$, tales que $Y \sqsubseteq Z$, si

$\sup _{X} Y \operatorname{y~sup}_{X} Z\left(\inf _{X} Y \operatorname{einf}_{X} Z\right)$ existen, entonces, se tiene que $\sup _{X} Y \preceq \sup _{X} Z$ $\left(\inf _{X} Y \preceq \inf _{X} Z\right)$.

\section{Demostración}

Considere $y \in Y$ y $z \in Z$. Como $Y \sqsubseteq Z$, se tiene que $y \wedge z \in Y$ y $y \vee z \in Z$, luego $y \preceq y \vee z \preceq \sup _{X} Z$, esto sucede para todo $y \in Y$. Así $\sup _{X} Y \preceq \sup _{X} Z$.

De manera análoga, se prueba que inf $\operatorname{in}_{X}\{Y\} \preceq y \wedge z \preceq z, \forall z \in Z$, entonces inf $\operatorname{in}_{X}\{Y\} \preceq$ $\inf _{X}\{Z\}$. 
A continuación, daremos una definición de una correspondencia (o multifunción). Este concepto será de gran importancia ya que, más adelante daremos una caracterización de los puntos de equilibrio en juegos no cooperativos basada en una correspondencia particular.

Definición 2.37. Una función cuyo rango está contenido en $\mathscr{P}(X)$, para algún conjunto $X$, es llamada correspondencia.

Definición 2.38. Una correspondencia $\mathrm{S}_{t}: T \rightarrow \mathscr{P}(X)$ se dice creciente (decreciente) en $t$, para $t \in T$, si:

a) El dominio $T$, es un conjunto parcialmente ordenado.

b) El rango, $\left\{S_{t}: t \in T\right\} \subset \mathscr{L}(X)$, con $X$ una látiz y $\mathscr{L}(X)$ parcialmente ordenado con relación $\sqsubseteq$.

c) Los conjuntos $S_{t}$ son crecientes (decrecientes), i.e. $t \preceq r \Rightarrow S_{t} \sqsubseteq S_{r}\left(S_{r} \sqsubseteq S_{t}\right)$.

Teorema 2.39. Sean $X$ y $T$ látices.

a) Si $S$ es una sublátiz de $X \times T$ entonces, la sección $S_{t}$ de $S$ en $t, t \in T$, es creciente en $t$ sobre la proyección $\Pi_{T} S$ de $S$ en $T$.

b) Si $T$ es una cadena, $S_{t} \subset X, \forall t \in T$, $\mathrm{S}_{t}$ es creciente en los $t$ con $S_{t} \neq \emptyset \mathrm{y}$ $S=\left\{(x, t): t \in T, x \in S_{t}\right\}$, entonces $S$ es una sublátiz de $X \times T$.

\section{Demostración}

a) Se probará que para $t, r \in \Pi_{T} S$, con $t \preceq r$, se tiene que $S_{t} \sqsubseteq S_{r}$.

Considere $t, r \in \Pi_{T} S$, tales que $t \preceq r$, y sean $a \in S_{t}, b \in S_{r}$, se tiene que

$$
(a, t) \wedge(b, r)=(a \wedge b, t) \quad \text { y } \quad(a, t) \vee(b, r)=(a \vee b, r)
$$

de esto que $a \wedge b \in S_{t}$ y $a \vee b \in S_{r}$, así los conjuntos $S_{t}$ con crecientes en $t \in \Pi_{T} S$.

b) Se probará que $a \vee b, a \wedge b \in S$, para cualesquiera $a, b \in S$.

Sean $(a, x)$ y $(b, y)$ en $S$, sin pérdida de generalidad, se puede suponer que $x \preceq y$, pues $T$ es una cadena, además $S_{x} \sqsubseteq S_{y}$, con $a \in S_{x}$ y $b \in S_{y}$. Luego, $a \wedge b \in S_{x}$ y $a \vee b \in S_{y}$, de aquí que $a \wedge b$ y $a \vee b$ estén en $S$ y se concluye que $S$ es una sublátiz. 
La siguiente observación se usa en la prueba de algunos resultados de la subsección 2.2.5.

Observación 2.40. Sean $X, Y$ conjuntos parcialmente ordenados, no vacíos. Considere $f: X \rightarrow \mathscr{P}(Y)$, si $f$ es una correspondencia creciente y $y \preceq x$ en $X$ entonces, para cualquier $s \in f(x)$ existe $t \in f(y)$ tal que, $t \preceq s$.

\subsubsection{Puntos fijos}

En esta parte definimos puntos fijos para funciones y correspondencias, además daremos algunas propiedades para cuando las correspondencias cumplen con ciertas características. Esta subsección es de las más importantes, ya que, los puntos de equilibrio en juegos no cooperativos se pueden ver como puntos fijos de la correspondencia de mejor respuesta.

Iniciaremos esta subsección con el concepto de punto fijo para una función y para una correspondencia.

Definición 2.41. Sea $f$ una función de un conjunto $X$ en sí mismo, si $x^{*} \in X$ satisface que $f\left(x^{*}\right)=x^{*}$ entonces, diremos que, $x^{*}$ es un punto fijo de $f$.

Similarmente, si $f: X \rightarrow \mathscr{P}(X)$ es una correspondencia tal que, para $x^{*} \in X, x^{*} \in$ $f\left(x^{*}\right)$, decimos que $x^{*}$ es un punto fijo de $f$.

Teniendo la definición de punto fijo en correspondencias, el siguiente resultado se tiene cuando el conjunto $X$ es una látiz completa, no vacía, y la correspondencia cumple algunas hipótesis sobre su rango e imagen.

Teorema 2.42. Sean $X$ una látiz completa no vacía y $Y: X \rightarrow \mathscr{L}(X)$ una correspondencia creciente, donde $\mathscr{L}(X)$ tiene el orden inducido por $\sqsubseteq$ y $Y(x)$ es una sublátiz subcompleta para cada $x \in X$, entonces:

a) El conjunto de puntos fijos de $Y$ en $X$ es no vacío, además, tiene elementos máximo y mínimo, dados por $\sup _{X}\{x \in X: Y(x) \cap[x, \infty) \neq \emptyset\}$ e ínf $X\{x \in X$ : $Y(x) \cap(-\infty, x] \neq \emptyset\}$, respectivamente.

b) El conjunto de puntos fijos de $Y$ en $X$ es una látiz completa y no vacía.

Ahora, para correspondencias crecientes cuyo dominio es un producto de conjuntos con ciertas propiedades, se tiene lo siguiente: 
Teorema 2.43. Sean $X$ una látiz completa, $T$ un conjunto parcialmente ordenado y $\mathrm{Y}: X \times T \rightarrow \mathscr{L}(X)$ una correspondencia creciente con $Y(x, t)$ una sublátiz subcompleta para cada $(x, t) \in X \times T$.

a) Para cada $t \in T$, la correspondencia Y tiene un máximo (mínimo) punto fijo.

b) El máximo (mínimo) punto fijo de $\mathrm{Y}$ es creciente en $T$.

c) Si también se supone que $\sup Y(x, t) \prec$ ínf $Y(x, s), \forall x \in X$ y $t \prec s$, entonces el máximo (mínimo) punto fijo de $Y$ es estrictamente creciente en $T$.

(la demostración puede verse en [21] página 39).

Cuando las imagenes de la correspondencia Y son singuletes, los resultados anteriores se pueden reescribir como:

Corolario 2.44. Sea $f: X \rightarrow X$, una función creciente de una látiz completa y no vacía, en sí misma.

a) El conjunto de puntos fijos de $f$ es no vacío y sus elementos máximo y mínimo se pueden describir como $\sup \{x \in X: x \preceq f(x)\}$ e ínf $\{x \in X: f(x) \preceq x\}$, respectivamente.

b) El conjunto de puntos fijos de $f$ es una látiz completa y no vacía.

(la demostración puede verse en [21] página 40).

Corolario 2.45. Sean $X$ una látiz completa y no vacía, $T$ un conjunto parcialmente ordenado y $f: X \times T \rightarrow X$ una función creciente, entonces:

a) La función $f$ tiene un máximo (mínimo) punto fijo, para cada $t \in T$.

b) El máximo (mínimo) punto fijo de $f$ es creciente en $T$.

c) Si además, $f$ es estrictamente creciente en $T$, para cada $x \in X$, entonces el máximo (mínimo) punto fijo de $f$ es estrictamente creciente en $T$.

(la demostración puede verse en [21] página 41).

\subsection{Funciones supermodulares}

En esta sección definimos las funciones supermodulares. Para esta parte debemos tomar mayor énfasis a estas funciones ya que para temas que abordamos en los siguientes capítulos la supermodularidad es una característica de las utilidades en los juegos supermodulares no cooperativos. 
Definición 2.46. Sean $X$ y $T$ conjuntos parcialmente ordenados y sea $f$ una función $f: \hat{S} \rightarrow \mathbb{R}$, con $\hat{S} \subset X \times T$. Si $f(x, t)-f(x, r)$ es creciente (decreciente, estrictamente creciente o estrictamente decreciente) en $x \in \hat{S}_{t} \cap \hat{S}_{r}$, para cualesquiera $t, r \in T$, con $r \prec t$, entonces diremos que $f$ tiene diferencias crecientes (decrecientes, estrictamente crecientes o estrictamente decrecientes).

Nota 2.47. La definición de diferencias crecientes es válida para ambas entradas de $f$ pues,

$$
f(x, t)-f(x, r) \leq f(y, t)-f(y, r) \Leftrightarrow f(y, r)-f(x, r) \leq f(y, t)-f(x, t) .
$$

Cuando el dominio de $f$ es subconjunto de un producto arbitrario de conjuntos parcialmente ordenados, la condición de diferencias crecientes se describe de la siguiente manera.

Observación 2.48. La función $f$ tiene diferencias crecientes cuando el dominio de $f$ es subconjunto de un producto arbitrario de conjuntos parcialmente ordenados, es decir, $f: X \rightarrow \mathbb{R}$, con $X \subset \prod_{\alpha \in A} X_{\alpha}$ y para cualesquiera $\alpha, \beta \in A, \alpha \neq \beta$, y cualquier $x_{\gamma} \in X_{\gamma}$, con $\gamma \in A \backslash\{\alpha, \beta\}$, $f$ tiene diferencias crecientes en los $\left(x_{\alpha}, x_{\beta}\right)$ pertenecientes a la sección de $X$ en $x_{\gamma}$.

Teorema 2.49. Dada la función $f: \mathbb{R}^{n} \rightarrow \mathbb{R}$, diferenciable en $\mathbb{R}^{n}$, ésta tiene diferencias crecientes si y sólo si $\frac{\partial f(x)}{\partial x_{i}}$ es creciente en $x_{j}$, para cualquier $x=\left(x_{1}, x_{2}, \ldots, x_{n}\right)$, con $j \neq i$. Equivalentemente, si $f$ tiene segunda derivada, se dice que tiene diferencias crecientes si y sólo si $\frac{\partial^{2} f(x)}{\partial x_{i} \partial x_{j}} \geq 0$, con $i \neq j$, para cualquier $x \in \mathbb{R}^{n}$.

\section{Demostración}

Por simplicidad, la prueba se hará para $n=2$, el caso general es análogo.

Se dice que la función $f$ tiene diferencias crecientes si, $f(\cdot, b)-f(\cdot, a)$ es una función creciente, con $b>a, a, b \in \mathbb{R}$; en otra palabras, si

$$
\frac{\partial}{\partial y}[f(y, b)-f(y, a)] \geq 0 \text { es decir, si } \frac{\partial f(y, b)}{\partial y}>\frac{\partial f(y, a)}{\partial y}
$$

Dicho de otro modo, si $\frac{\partial f(y, a)}{\partial y}$ es una función creciente en $a$, lo cual equivale a decir que $\frac{\partial^{2} f(y, a)}{\partial y \partial a} \geq 0$ 
Definición 2.50. Sea $X$ una látiz y consideremos una función $f$, tal que, $f: X \rightarrow \mathbb{R}$, diremos que $f$ es supermodular en $X$ si, para cualesquiera $x, y \in X, f$ se cumple:

$$
f(x)+f(y) \leq f(x \vee y)+f(x \wedge y)
$$

Si la desigualdad es estricta, diremos que $f$ es estrictamente supermodular. Similarmente, diremos que $f$ es submodular en $X$ si $-f$ es supermodular.

Una función que es supermodular y submodular es llamada valuación.

El siguiente resultado, cuya prueba se puede revisar en [21] (página 44, Teorema 2.6.1), caracteriza las funciones con diferencias crecientes en sublátices.

Teorema 2.51. Sean $A$ un conjunto de índices, $X_{\alpha}$ una látiz, para cada $\alpha \in A$, y $X \subset \prod_{\alpha \in A} X_{\alpha}$ una sublátiz. Si $f$ es supermodular en $X$, entonces $f$ tiene diferencias crecientes en $X$.

A continuación, mostramos dos resultados que identifican la supermodularidad en términos de diferencias crecientes cuando el dominio de la función tiene ciertas características; seguido de esto, se muestran algunos ejemplos de funciones supermodulares.

Teorema 2.52. Sea $X_{i}$ una látiz, para $i=1, \ldots, n$. Si $f: \prod_{i=1}^{n} X_{i} \rightarrow \mathbb{R}$ es una función con diferencias crecientes y supermodular en cada entrada fija, entonces $f$ es supermodular en $\prod_{i=1}^{n} X_{i}$.

La demostración del Teorema 2.52 se encuentra en [21] (página 45, Teorema $2.6 .2)$.

Corolario 2.53. Considere $N=\{1, \ldots, n\}$, con $n \in \mathbb{N}, X_{i}$ una cadena para cada $i \in N$ y $f$ una función con diferencias crecientes en $\prod_{i=1}^{n} X_{i}$, entonces $f$ es supermodular en $\prod_{i=1}^{n} X_{i}$.

Observación 2.54. El resultado anterior no es cierto cuando $N$ no es finito. (ver [21], página 45)

Ejemplo 2.55. Las siguientes funciones son supermodulares.

1. Sea $X$ una cadena, cualquier función $f: X \rightarrow \mathbb{R}$ es una valuación. 
2. La función $f\left(x_{1}, x_{2}\right)=x_{1} x_{2}$ es supermodular en $\mathbb{R}^{2}$.

3. La función $f\left(x_{1}, \ldots, x_{n}\right)=\operatorname{mín}\left\{\alpha_{i} x_{i}: i=1, \ldots, n\right\}$, con $\alpha_{i} \geq 0$, para $i=1, \ldots, n$, es supermodular en $\mathbb{R}^{n}$.

\subsubsection{Conjunto de soluciones óptimas}

En esta parte definiremos el conjunto de soluciones óptimas de una función. Este concepto lo usaremos al momento de definir la correspondencia de mejor respuesta en juegos supermodulares no cooperativos.

Definición 2.56. Dado el problema de maximizar una función real valuada, $f: X \rightarrow$ $\mathbb{R}$, el conjunto de soluciones óptimas de $f$ se define como:

$$
\operatorname{argmax}_{x \in X} f(x)=\{x \in X: f(y) \leq f(x), \forall y \in X\} .
$$

Los resultados que se enunciarán a continuación, serán de utilidad para asegurar la existencia de equilibrios en juegos supermodulares no cooperativos.

Teorema 2.57. Sean $X$ una látiz y $f: X \rightarrow \mathbb{R}$ una función supermodular, entonces $\operatorname{argmax}_{x \in X} f(x)$ es una sublátiz de $X$.

Demostración

Sean $x, y \in \operatorname{argmax}_{x \in X} f(x)$, como $f$ es supermodular, se tiene que:

$$
f(x)+f(y) \leq f(x \wedge y)+f(x \vee y),
$$

así,

$$
0 \leq f(x)-f(x \wedge y) \leq f(x \vee y)-f(y) \leq 0,
$$

donde la primera y la última desigualdad se dan porque $x$ y $y$ maximizan la función $f$, se sigue que $x \vee y$ y $x \wedge y$ están en $\operatorname{argmax}_{x \in X} f(x)$.

Corolario 2.58. Sea $X \neq \emptyset$ una látiz y $f: X \rightarrow \mathbb{R}$ una función supermodular. 
a) Si $X$ es finita, entonces $\operatorname{argmax}_{x \in X} f(x)$ es una sublátiz subcompleta, no vacía, de $X$.

b) Si $X$ es una sublátiz compacta de $\mathbb{R}^{n}$ y $f$ es semicontinua superiormente en $X$, entonces $\operatorname{argmax}_{x \in X} f(x)$ es una sublátiz de $\mathbb{R}^{n}$, subcompleta, compacta y no vacía.

Teorema 2.59. Sean $X$ y $Y$ látices, $\hat{S} \subset X \times Y$ una sublátiz y $f: \hat{S} \rightarrow \mathbb{R}$ una función supermodular. Si $g(y)=\sup _{x \in \hat{S}_{y}} f(x, y)$ es finito en $\Pi_{Y} \hat{S}$, entonces $g$ es supermodular.

\section{DEMOSTRACIÓN}

Considere $y, z \in \Pi_{Y} \hat{S}$ y sean $a \in \hat{S}_{y}$ y $b \in \hat{S}_{z}$. La función $f$ es supermodular, de esto que

$$
f(a, y)+f(b, z) \leq f(a \vee b, y \vee z)+f(a \wedge b, y \wedge z)
$$

tomando supremos, se tiene que

$$
g(y)+g(z) \leq g(y \vee z)+g(y \wedge z)
$$

y se concluye lo deseado.

Los siguientes resultados dan condiciones suficientes para que el conjunto de soluciones óptimas, $\operatorname{argmax}_{x \in S_{t}} f(x, t)$, sea creciente.

Lema 2.60. Sean $X$ una látiz, $T$ un conjunto parcialmente ordenado y $\hat{S}_{t} \subset X$, para cada $t \in T$. Si los conjuntos $\hat{S}_{t}$ son crecientes en $t \mathrm{y}$,

$$
f(x, t)+f(y, r) \leq f(x \wedge y, t)+f(x \vee y, r),
$$

para cualesquiera $t, r \in T$, con $t \preceq r$ y $x \in \hat{S}_{t}, y \in \hat{S}_{r}$, entonces $\operatorname{argmax}_{x \in \hat{S}_{t}} f(x, t)$ es creciente en $t \in\left\{t \in T: \operatorname{argmax}_{x \in \hat{S}_{t}} f(x, t) \neq \emptyset\right\}$.

\section{Demostración}

Se probará que, para $x \in \operatorname{argmax}_{a \in \hat{S}_{t}} f(a, t)$ y $y \in \operatorname{argmax}_{a \in \hat{S}_{r}} f(a, r)$, con $t \preceq r$, en $\left\{t \in T: \operatorname{argmax}_{x \in \hat{S}_{t}} f(x, t) \neq \emptyset\right\}$, se cumple que $x \wedge y \in \operatorname{argmax}_{a \in \hat{S}_{t}} f(a, t)$ y 
$x \vee y \in \operatorname{argmax}_{a \in \hat{S}_{r}} f(a, r)$.

El conjunto de soluciones óptimas de $f$ es una sublátiz, para cada $t \in T$. Considere $t, r \in\left\{t \in T: \operatorname{argmax}_{x \in \hat{S}_{t}} f(x, t) \neq \emptyset\right\}$, tales que $t \preceq r$. Ahora, tome $x \in$ $\operatorname{argmax}_{a \in \hat{S}_{t}} f(a, t)$ y $y \in \operatorname{argmax}_{a \in \hat{S}_{r}} f(a, r)$. Como $\hat{S}_{t} \sqsubseteq \hat{S}_{r}$, se tiene que $x \wedge y \in \hat{S}_{t} \mathrm{y}$ $x \vee y \in \hat{S}_{r}$. Luego, de 2.1 se sigue que:

$$
0 \leq f(x, t)-f(x \wedge y, t) \leq f(x \vee y, r)-f(y, r) \leq 0
$$

De esto que $f(x, t)=f(x \wedge y, t)$ y $f(x \vee y, r)=f(y, r)$. Así $x \wedge y \in \operatorname{argmax}_{a \in \hat{S}_{t}} f(a, t)$ y $x \vee y \in \operatorname{argmax}_{a \in \hat{S}_{r}} f(a, r)$.

Teorema 2.61. Sean $X$ una látiz, $T$ un conjunto parcialmente ordenado y para cada $t \in T, \hat{S}_{t} \subset X$, subconjunto creciente en $t$. Considere $f(x, t)$ una función supermodular en $X$, con diferencias crecientes en $X \times T$. Entonces $\operatorname{argmax}_{x \in \hat{S}_{t}} f(x, t)$ es creciente en $t \in\left\{t \in T: \operatorname{argmax}_{x \in \hat{S}_{t}} f(x, t) \neq \emptyset\right\}$.

Teorema 2.62. Sean $X$ y $T$ látices y $\hat{S} \subset X \times T$, una sublátiz. Tome $\hat{S}_{t}$ la sección de $\hat{S}$ en $t \in T$ y $f$ una función supermodular en $\hat{S}$, entonces $\operatorname{argmax}_{x \in \hat{S}_{t}} f(x, t)$ es creciente para $t \in\left\{t \in T: \operatorname{argmax}_{x \in \hat{S}_{t}} f(x, t) \neq \emptyset\right\}$.

Teorema 2.63. Si se cumplen las hipótesis del Teorema 2.61 o del Teorema 2.62 y cada $K_{t} \neq \emptyset$, con $S_{t}$ finito o subconjunto compacto de $\mathbb{R}^{m}$ y $f$ es semicontinua superiormente, para cada $t \in T$, entonces $\operatorname{argmax}_{x \in K_{t}} f(x, t)$ es una sublátiz no vacía de $X$ y sus elementos máximo y mínimo son crecientes en $t \in T$.

Las pruebas de los Teoremas 2.61, 2.62, 2.63, se encuentran en [21], páginas 77-78, Teoremas 2.8.1, 2.8.2, 2.8.3.

\subsection{Definición de juego supermodular}

Definición 2.64. Para cada $i \in N$, sea $E_{i}$ el conjunto de estrategias admisibles del jugador $i$, con $\left|E_{i}\right|=m_{i} \in \mathbb{N}$. $E \subset \prod_{i=1}^{n} E_{i}$, es llamado conjunto de perfiles de estrategias admisibles, los perfiles de estrategias (estrategias conjuntas) son $\mathbf{x}=\left(x_{1}, \ldots, x_{n}\right)$, donde $x_{i}$, la estrategia del jugador $i$, es un vector de tamaño $m_{i}, \forall i \in N$. 
Ahora, se puede definir lo que es un juego no cooperativo de la siguiente manera.

Definición 2.65. Un juego no cooperativo es una triada $\left(N, E,\left\{U_{i}: i \in N\right\}\right)$, donde $N \neq \emptyset$ es el conjunto de jugadores, $E$ es el conjunto de perfiles estrategias admisibles y $\left\{U_{i}: i \in N\right\}$ es la colección de funciones de pago correspondientes a cada jugador, con $U_{i}: E \rightarrow \mathbb{R}$.

Nota 2.66. Este trabajo se enfoca en los juegos con un número finito de jugadores entonces, el conjunto de jugadores se considera de la forma $N=\{1, \ldots, n\}, n \in \mathbb{N}$.

A continuación, se introduce algo de notación para facilitar el enunciado de resultados posteriores.

Nota 2.67. Dado un perfil de estrategias $x \in E$ y $y_{i} \in E_{i}$, entonces:

a) $x_{-i}$, es el vector de estrategias correspondientes a los jugadores en el conjunto $N \backslash\{i\}$.

b) $\left(x ; y_{i}\right)$, es el perfil de estrategias donde la estrategia del $i$-ésimo jugador se cambia a $y_{i}$ y los demás jugadores mantienen su estrategia en $x$.

c) $E_{i}\left(x_{-i}\right)=\left\{y_{i}:\left(x_{-i} ; y_{i}\right) \in E\right\}$, denota el conjunto de estrategias admisibles para el jugador $i$, dadas las estrategias $x_{-i}$ de los demás jugadores, i.e. $E_{i}\left(x_{-i}\right)$ es la sección de $E$ en $x_{-i}$.

d) $E_{-i}=\left\{x_{-i}: E_{i}\left(x_{-i}\right) \neq \emptyset\right\}$, la colección de vectores $x_{-i}$ tales que, existe $y_{i} \in E_{i}$ con $\left(x_{-i} ; y_{i}\right) \in E$. En otras palabras, $E_{-i}$ es la proyección de $E$ en las estrategias de todos los jugadores distintos de $i$.

e) $E(x)=\left(\prod_{i=1}^{n} E_{i}\left(x_{-i}\right)\right) \cap E$

Observación 2.68. $E=\prod_{i=1}^{n} E_{i}$ si y sólo si $E(x)=E$, para todo perfil de estrategias admisibles $\mathbf{x} \in E$.

Otro concepto de suma importancia en la Teoría Juegos es el de punto de equilibrio, que ahora se presenta:

Definición 2.69. Un perfil de estrategias admisibles $x$ es llamado punto de equilibrio si

$$
U_{i}\left(x_{-i} ; y_{i}\right) \leq U_{i}(x), \forall y_{i} \in E_{i}\left(x_{-i}\right)
$$


en otras palabras, dado un punto de equilibrio, ningún jugador puede incrementar su pago cambiando de estrategia, si las estrategias de los demás jugadores se mantienen fijas.

Con las definiciones y notación previa, se procede a definir los juegos supermodulares no cooperativos.

Definición 2.70. Un juego no cooperativo $\left(N, E,\left\{U_{i}: i \in N\right\}\right)$ se dice supermodular si, $E \subset \mathbb{R}^{m}$ es una sublátiz, la función de pago $U_{i}\left(x_{-i} ; y_{i}\right)$ es supermodular en $y_{i} \in E_{i}$, para cada $x_{-i} \in E_{-i}, \forall i \in N ;$ y $U_{i}\left(x_{-i} ; y_{i}\right)$ tiene diferencias crecientes en $\left(x_{-i} ; y_{i}\right)$.

Las definiciones 2.71 y 2.73 , nos serán de utilidad para caracterizar los puntos de equilibrio en los juegos supermodulares no cooperativos.

Definición 2.71. Sea $x_{-i} \in E_{-i}$, la correspondencia de mejor respuesta para el jugador $i$, es el conjunto de estrategias óptimas para el jugador $i$, cuando las estrategias de los demás jugadores están dadas por $x_{-i}$. Dicha correspondencia se puede describir como,

$$
\Lambda_{i}\left(x_{-i}\right)=\operatorname{argmax}_{y_{i} \in E_{i}\left(x_{-i}\right)} U_{i}\left(x_{-i} ; y_{i}\right)
$$

Nota 2.72. El conjunto de estrategias óptimas para el jugador $i$, cuando las estrategias de los demás jugadores están dadas por $x_{-i}$, se puede ver como el conjunto de soluciones óptimas de $U_{i}\left(x_{-i} ; \cdot\right)$.

Definición 2.73. Sean $\mathbf{x} \in E$ y $\mathbf{y} \in E(\mathbf{x})$, considere $G(\mathbf{y}, \mathbf{x})=\sum_{i \in N} U_{i}\left(x_{-i} ; y_{i}\right)$. Para cada perfil de estrategias admisibles, $\mathbf{x} \in E$, la correspondencia de mejor respuesta conjunta está dada por :

$$
\Lambda(\mathbf{x})=\operatorname{argmax}_{\mathbf{y} \in S(\mathbf{x})} G(\mathbf{y}, \mathbf{x})
$$

este es el conjunto de todos los perfiles de estrategias admisibles tales que, la estrategia del jugador $i$ es admisible dada $x_{-i}$ y la suma de los pagos de todos los jugadores es maximizada, pues deciden cambiar a la estrategia correspondiente en $\mathbf{y}$, en lugar de mantener la estrategia $x_{i}$, y esto sucede para todo $i$ en $N$. 


\subsection{Existencia de puntos de equilibrio}

En esta sección presentamos resultados los cuales nos aseguran la existencia de puntos de equilibrio en juegos supermodulares no cooperativos, que es una manera muy similar que obtener las condiciones necesarias en el teorema de punto fijo de Kakutani (ver [11]) (generalizacion del Teorema de Brouwer).

Lema 2.74. Considere un juego supermodular $\left(N, E,\left\{U_{i}: i \in N\right\}\right)$ en el cual, el conjunto de estrategias admisibles $S$, es compacto y no vacío, la función de pago $U_{i}\left(x_{-i} ; y_{i}\right)$ es semicontinua superiormente en $y_{i} \in E_{i}\left(x_{-i}\right), \forall x_{-i} \in E_{-i}, i \in N$. Entonces

a) Para cada jugador $i$, la correspondencia de mejor respuesta $\Lambda\left(x_{-i}\right)$ es creciente en $x_{-i} \in E_{-i}$; además, tiene elementos máximo y mínimo y estos son funciones crecientes de $E_{-i}$ en $E_{i}$. El conjunto $\Lambda_{i}\left(x_{-i}\right)$ de mejores respuestas para cada jugador $i$ es una sublátiz subcompleta, compacta y no vacía de $\mathbb{R}^{m_{i}}$, para todo $x_{-i} \in E_{-i}$.

b) La correspondencia de mejor respuesta conjunta $\Lambda(\mathbf{x})$ es creciente para $\mathbf{x} \in$ $S$. Los elementos máximo y mínimo de dicha correspondencia son funciones crecientes de $E$ en $E$. El conjunto $\Lambda(\mathbf{x})$ de perfiles de mejor respuesta, es una sublátiz subcompleta, compacta y no vacía de $\mathbb{R}^{m}$, para cada $\mathbf{x} \in S$.

Ahora, se presentará un resultado de J. Nash (ver [14]), que nos asegura la existencia de equilibrios en juegos con conjunto de jugadores y conjunto de perfiles de estrategias finitos.

Teorema 2.75. Todo juego con número finito de jugadores y conjunto de perfiles de estrategias finito tiene un punto de equilibrio.

Teniendo este resultado, para los juegos no cooperativos se tiene el siguiente lema, que caracteriza los puntos de equilibrio como puntos fijos de la correspondencia de mejor respuesta conjunta.

Lema 2.76. El conjunto de puntos de equilibrio de un juego no cooperativo $\left(N, E,\left\{U_{i}\right.\right.$ : $i \in N\}$ ) es idéntico al conjunto de puntos fijos de la correspondencia de mejor respuesta conjunta $\Lambda(\mathbf{x}), \mathbf{x} \in S$. 


\section{DEMOSTRACIÓN}

Se probará que los conjuntos de puntos de equilibrio y el conjunto de puntos fijos son iguales.

Sea $\mathbf{x} \in E$ un punto fijo de la correspondencia de mejor respuesta conjunta. Considere al jugador $i$ y $y_{i} \in E_{i}\left(x_{-i}\right)$, se tiene que $\mathbf{x}^{\prime}=\left(x_{-i}, y_{i}\right) \in E(\mathbf{x})$, luego,

$$
0 \leq G(\mathbf{x}, \mathbf{x})-G\left(\mathbf{x}^{\prime}, \mathbf{x}\right)=U_{i}(\mathbf{x})-U_{i}\left(x_{-i} ; y_{i}\right)
$$

y esto sucede para cualquier $i \in N$, pues el $i$ elegido fue arbitrario, de aquí que $\mathbf{x}$ sea un punto de equilibrio.

Ahora, tómese $\mathbf{x}$ un punto de equilibrio y $\mathbf{y} \in E(\mathbf{x})$, entonces $y_{i} \in E_{i}\left(x_{-i}\right), \forall i \in N$. Como $\mathbf{x}$ es un punto de equilibrio, $U_{i}(\mathbf{x}) \geq U_{i}\left(x_{-i} ; y_{i}\right)$, luego,

$$
\begin{aligned}
0 \leq \sum_{i \in N}\left(U_{i}(x)-U_{i}\left(x_{-i} ; y_{i}\right)\right) & =\sum_{i \in N} U_{i}(x)-\sum_{i \in N} U_{i}\left(x_{-i} ; y_{i}\right) \\
& =G(x, x)-G(y, x) .
\end{aligned}
$$

Luego, $G(x, x) \geq G(y, x)$, con $y$ elegido de manera arbitraria en $E(x)$, así $x$ maximiza la función $G$, en otras palabras, $x$ pertenece al conjunto $\Lambda(x)$, es decir, es un punto fijo de la correspondencia de mejor respuesta conjunta.

Contando con lo anterior, si se agregan algunas características al conjunto de perfiles de estrategias y a las funciones de pago, el siguiente resultado nos asegura la existencia de puntos de equilibrio en los juegos supermodulares no cooperativos.

Observación 2.77. Punto fijo de Kakutani [11]

Sea $E \subset \mathbb{R}^{m}$ un conjunto compacto y convexo, además supongamos un mapeo $\psi: S \rightarrow E$ superiormente semicontinuo, convexo y que cumple que $\forall X \in E, \psi(X)$ es no vacío. Entonces, existe $\hat{X} \in S$ tal que $\hat{X} \in \psi(\hat{X})$. (ver demostración en [11] o en [24], página 222)

Teorema 2.78. Dado un juego supermodular $\left(N, E,\left\{U_{i}: i \in N\right\}\right)$, con conjunto de perfiles de estrategias admisibles $E$, no vacío y compacto, cuya función de pago 
$U_{i}\left(x_{-i} ; y_{i}\right)$ es semicontinua superiormente en $y_{i} \in E_{i}\left(x_{-i}\right)$, para $x_{-i} \in E_{-i}, \forall i \in N$. Entonces, el conjunto de puntos de equilibrio es una látiz completa y no vacía, que tiene elementos máximo y mínimo.

\section{DEMOSTRACIÓN}

Recuerde que el conjunto de puntos de equilibrio de un juego no cooperativo es igual al conjunto de puntos fijos de la correspondencia de mejor respuesta conjunta. Ahora, observe que:

- $E \subset \mathbb{R}^{m}$ es una sublátiz no vacía y compacta, así, por el Teorema 2.32, $E$ es una sublátiz subcompleta. De esto que $E$ sea una látiz completa.

- Del Lema 2.74, se tiene que la correspondencia de mejor respuesta conjunta es una sublátiz compacta de $\mathbb{R}^{m}$ así, $\Lambda(x)$ es una sublátiz subcompleta.

Teniendo estas observaciones, por el Teorema 2.42, se sigue que el conjunto de puntos de equilibrio de la correspondencia de mejor respuesta conjunta es una látiz no vacía y completa, de la completez de este conjunto, se puede concluir que tiene elementos máximo y mínimo.

\subsection{Algoritmo de optimización Round-Robin}

Dado un juego no cooperativo $\left(N, E,\left\{U_{i}: i \in N\right\}\right)$, siguiendo los pasos que se enuncian a continuación, se genera una sucesión (finita o infinita) de perfiles de estrategias admisibles.

a) Si $E$ tiene un elemento mínimo, $\operatorname{lnf}(E)$, haga $x^{0,0}=\operatorname{lnf}(E)$. En caso contrario, se detiene.

b) Dado $x^{i, j} \in S$, con $i, j \in \mathbb{N}$ e $j<n$, considere $x^{i, j+1}=\left(x_{j+1}^{k, j+1}, x_{-(j+1)}^{i, j}\right)$, donde $x_{j+1}^{i, j+1}$ es el elemento mínimo de la correspondencia de mejor respuesta $\Lambda_{j+1}\left(x_{-(j+1)}^{i, j}\right)$, si este elemento existe. En otro caso, deténgase.

c) Se incrementa $j=j+1$, si $j=n$ y $x^{i, n}$ se ha generado para algún $i$, considere $x^{i+1,0}=x^{i, n}$, aumente $i=i+1$ y haga $j=0$. Regrese al paso b). 
Los siguientes resultados, aseguran que el algoritmo de Round-Robin genera equilibrios en juegos supermodulares no cooperativos e incluso proveen una cota para el número de iteraciones necesarias para encontrar un equilibrio cuando el conjunto de perfiles de estrategias admisibles es finito.

Teorema 2.79. Dado un juego supermodular $\left(N, E,\left\{U_{i}: i \in N\right\}\right)$, con conjunto de perfiles de estrategias admisibles no vacío y compacto, cuyas funciones de utilidad, $U_{i}\left(x_{-i} ; y_{i}\right)$, son semicontinuas superiormente en $y_{i} \in E_{i}\left(x_{-i}\right)$, para cada $x_{-i} \in E_{-i}$. Se tiene que:

a) El algoritmo de Round-Robin no se detiene en los pasos a) ó b). Además, genera una sucesión infinita $x^{k, i}$, creciente en $k$ e $i$, para $k=0,1, \ldots, i=0, \ldots, n$. De esto que exista un perfil de estrategias admisibles $x^{*} \in S$ tal que, $\lim _{k \rightarrow \infty} x^{k, i}=$ $x^{*}$, para $i=0, \ldots, n$.

b) Si en la sucesión generada por el algoritmo aparece un perfil de estrategias $n$ veces consecutivas, entonces ese perfil es un punto de equilibrio.

c) Si en alguna iteración se genera un punto de equilibrio, el algoritmo generará el mismo punto en cualquier iteración posterior.

d) Si además, el conjunto de perfiles de estrategias es finito y para cada conjunto de estrategias admisibles $, E_{i}, q_{i}$ es una cota superior de la cardinalidad de cualquier cadena contenida en dicho conjunto, entonces el algoritmo genera un punto de equilibrio en a lo más $(n-1)\left(\sum_{i=1}^{n} q_{i}\right)-n^{2}+n+1$ iteraciones.

La demostración del teorema anterior se puede revisar en [21] (página 187, Teorema 4.3.1).

\subsection{Conclusiones}

En este capítulo describimos las propiedades de los juegos supermodulares a partir de la definición de las látices y sublátices, desarrollando propiedades topológicas en estos juegos y describiendo lo que son las funciones supermodulares, junto con el conjunto de las soluciones óptimas para los juegos supermodulares y las condiciones necesarias para la existencia de puntos de equilibrio en éstos. Se concluye este capítulo con un algortimo que nos garantiza cómo encontrar dichos puntos. Este algoritmo es conocido como el algoritmo de Round Robin. 


\section{Capítulo 3}

\section{Juegos $S$-modulares y Ejemplos de Sistemas de Espera}

\subsection{Introducción}

Este capítulo está basado en [1], [2]. [4], [5], [8], [12], [20], [22], [24], que serán usados como referencia para el desarrollo de los juegos $S$-modulares con aplicaciones específicamente en las colas en Tándem sin salidas, donde comenzaremos la temática de un juego $S$-modular con sus definiciones, después abordaremos ejemplos para mostrar como resolver problemas de colas en Tándem sin salidas con las debidas suposiciones para obtener un equilibrio de Nash y donde desarrollaremos las hipótesis que deben hacerse para llegar a tener existencia en equilibrios de Nash en estos juegos, al final desarrollaremos un algoritmo con el cual a través de debidas hipótesis obtendremos unicidad en este tipo de juegos.

\subsection{Algoritmo $I$}

En el control óptimo de colas se desarrolló la noción de $S$-modularidad que trata la supermodularidad y la submodularidad simultáneamente, esto permite a la función objetivo ser supermodular con respecto a algunas variables y submodular con respecto a otras variables.

En esta sección empezaremos considerando el caso donde solamente hay dos jugadores, donde denotaremos con $i, i=1,2$ el número de jugadores en el juego. También denotaremos con $x_{i}$, la estrategia del jugador $i$ y $S_{i}$ corresponderá al espacio de estrategia del jugador $i$, ésta será una sublátiz compacta de dimensión $d_{i}$, empezaremos 
considerando el caso donde $d_{i}=1$. Haremos referencia con $x=\left(x_{1}, x_{2}\right)$ al perfil de estrategia de los jugadores, después se analizará un sistema con n jugadores y analizaremos los equilibrios por medio de la Teoría de Colas clásica, abordando unas aplicaciones de esta teoría.

Definición 3.1. El espacio de todos los perfiles de estrategia factibles están definidos de la siguiente manera:

$$
\tilde{S}:=\left\{x=\left(x_{1}, x_{2}\right)\right\}=\tilde{S}_{1} \times \tilde{S}_{2}
$$

Definición 3.2. Sea $f_{i}\left(x_{1}, x_{2}\right)$ la función de pago del jugador $i$, donde el jugador $i$ maximiza la función $f_{i}$ con respecto a $x_{j}$, dado que $i \neq j$.

Definición 3.3. Un perfil de estrategia, $x^{*}=\left(x_{1}^{*}, x_{2}^{*}\right)$, es denominado un equilibrio de Nash, si cumple las siguientes condiciones:

$$
\begin{aligned}
& f_{1}\left(x_{1}^{*}, x_{2}^{*}\right) \geq f_{1}\left(x_{1}, x_{2}^{*}\right), \forall x_{1} \in \tilde{S}_{1}, \\
& f_{1}\left(x_{1}^{*}, x_{2}^{*}\right) \geq f_{1}\left(x_{1}^{*}, x_{2}\right), \forall x_{2} \in \tilde{S}_{2} .
\end{aligned}
$$

De manera general, asumiremos que cada función de pago $f_{i}$ es continua en $x \in \tilde{S}$. Esto junto con la compacidad del espacio de estrategia, asegura la existencia de un maximizador, $x_{i}^{*} \in \tilde{S}_{i}$, de $f_{i}$, dado $x_{j} \in S_{j}, j \neq i$ (de hecho para la existencia de un maximizador, la semicontinuidad superior más débil de $f_{i}$ en $x_{i} \in \tilde{S}_{i}$ es suficiente).

Definición 3.4. Si hacemos $x_{1}^{*}\left(x_{2}\right)=\operatorname{argmax}_{x_{1} \in S_{1}} f\left(x_{1}, x_{2}\right)$ (esto es $x_{1}^{*}\left(x_{2}\right)$ es el maximizador de $f\left(x_{1}, x_{2}\right)$ dada la estrategia $\left.x_{2}\right)$, entonces este maximizador es creciente en $x_{2}$ de tal forma que si tenemos $x_{2} \leq x_{2}^{\prime} \Rightarrow x_{1}^{*}\left(x_{2}\right) \leq x_{1}^{*}\left(x_{2}^{\prime}\right)$

Observación 3.5. En aplicaciones, es a menudo conveniente permitir que el espacio de estrategia de cada jugador dependa del perfil de estrategia.

Definición 3.6. El espacio de estrategia del jugador $i$, dada la estrategia $x_{j}$ del jugador $j$, denotaremos por $\tilde{S}_{i}\left(x_{j}\right)$, con $i \neq j$. 


\subsubsection{Monotonicidad, continuidad y estabilidad}

Para definir de manera más general los equilibrios de Nash en 3.3, modificaremos los espacios de estrategia para exigir que se mantengan las desigualdades para toda $x_{i} \in \tilde{S}_{i}\left(x_{j}^{*}\right)$ con $i \neq j$ respectivamente. Asumiremos que $\tilde{S}_{1}\left(x_{2}\right)$ es un sublátiz compacto, para cada $x_{2}$ e igualmente para $\tilde{S}_{2}\left(x_{1}\right)$, además de establecer las siguientes condiciones:

Definición 3.7. Monotonicidad:

Si $x_{2} \leq x_{2}^{\prime} \Rightarrow \tilde{S}_{1}\left(x_{2}\right) \prec \tilde{S}_{1}\left(x_{2}^{\prime}\right)$, y $x_{1} \leq x_{1}^{\prime} \Rightarrow \tilde{S}_{2}\left(x_{1}\right) \prec \tilde{S}_{2}\left(x_{1}^{\prime}\right)$ esta propiedad es conocida como la propiedad ascendente que es un mapeo de un punto a un conjunto

( $A \prec B$ si para cualquier $a \in A$ y $b \in B$ se tiene que $a \wedge b \in A$ y $a \vee b \in B$. Para la propiedad descendente)

Definición 3.8. Continuidad:

Para definir la continuidad se tiene que si $x_{1}^{k} \rightarrow x_{1}^{*}$ para toda $k \in \mathbb{N}$ y si $x_{2}^{*} \in \tilde{S}_{2}\left(x_{1}^{*}\right)$, ésto implica la existencia de una sucesión $\left\{x_{2}^{k}\right\}$, tal que $x_{2}^{k} \in \tilde{S}_{2}\left(x_{1}^{k}\right)$ para toda $k$ y $x_{2}^{k} \rightarrow x_{2}^{*}$. De manera similar se define la continuidad para $\tilde{S}_{1}$.

\subsubsection{Estabilidad}

En esta subsección estableceremos la definición y condiciones en las cuales consideraremos la estabilidad de un conjunto, entre las cuales podemos hablar de estabilidad local o global.

Primero estableceremos las definiciones indicadas para poder desarrollar el concepto de estabilidad de la manera más general en equilibrios de Nash, comenzando por definir algunos conceptos básicos en la Teoría de Juegos.

Entonces la forma normal del juego es descrita por $\{U, V\}$ y $\left\{f_{1}, f_{2}\right\}$, donde asumiremos que $U$ y $V$ son espacios de Hilbert apropiados con productos interno $\langle,\rangle_{U} \mathrm{y}\langle,\rangle_{V}$, respectivamente.

Definición 3.9. Un equilibrio de Nash se dice estable (o globalmente estable) si puede ser obtenido como límite desde cualquier perfil de estrategia inicial.

Por lo tanto de aquí en adelante diremos que un equilibrio de Nash es localmente estable, si el equilibrio puede ser alcanzado desde cualquier perfil inicial dentro de alguna pequeña vecindad del punto de equilibrio. 


\subsubsection{Algoritmo $I$ para funciones $S$-modulares}

Retomando la notación que hemos llevado hasta el momento, de nuevo donde $i$ será la referencia de los jugadores, que en este caso, únicamente se consideraran dos jugadores, $i=1,2$. Con el superíndice indicaremos los pasos en el juego. Supongamos inicialmente el paso 0 y esto reflejaremos en el perfil de estrategia de los jugadores como $\left(x_{1}^{0}, x_{2}^{0}\right)$. En el paso 1, el jugador encuentra $x_{1}^{1}$ para maximizar su función de utilidad $f_{1}$ (dado $x_{2}^{0}$ ) y obtenemos el perfil de estrategia de los jugadores $\left(x_{1}^{1}, x_{2}^{0}\right)$ y después el jugador 2 encontrará $x_{2}^{1}$ para maximizar su función de utilidad $f_{2}$, dada la estrategia actualizada $x_{1}^{1}$ del jugador 1 , esto se repite en los siguientes pasos de manera sucesiva, este procedimiento describe el algoritmo I para dos jugadores.

Teorema 3.10. Consideremos un juego bipersonal y supongamos dos funciones de pago supermodulares (submodulares) y continuas, con los espacios de estrategia satisfaciendo las propiedades ascendentes (o propiedades descendentes), además de considerar que los espacios de estrategias deben ser continuos, compactos y sublátices, entonces las estrategias de los dos jugadores $\left\{x_{1}^{k}\right\}$ y $\left\{x_{2}^{k}\right\}$, convergen monotónicamente (en $k \geq 1$ ) a un equilibrio de Nash, desde cualquier pefril de estrategia.

\section{Demostración}

Sea $\tilde{S}_{i}\left(x_{j}\right)$, con $i=1,2, i \neq j$ espacios de estrategia que a la vez sean, sublatices compactos que cumplen la propiedad ascendente y la continuidad, debido a la propiedad monótona en maximizar las funciones de pago supermodulares, cuando seguimos el algoritmo I, claramente, $\left\{x_{1}^{k}\right\}$ y $\left\{x_{2}^{k}\right\}$ son dos sucesiones monótonas, en particular pueden ser 2 sucesiones ya sea ambas crecientes o ambas funciones decrecientes en $k \geq 1$. En caso de que ambas funciones $f_{1}$ y $f_{2}$ fueran submodulares en $\left(x_{1}, x_{2}\right)$, el único cambio es que $\left\{x_{1}^{k}\right\}$ y $\left\{x_{2}^{k}\right\}$ serán monótonas en dirección opuesta cuando $k \geq 1$ y en este caso el único cambio será pedir que el espacio de estrategia de los jugadores cumplan la propiedad descendente.

Supongamos que $x_{1}^{k} \rightarrow x_{1}^{*}$ y $x_{2}^{k} \rightarrow x_{2}^{*}$ y por lo anterior hemos mostrado que $\left(x_{1}^{*}, x_{2}^{*}\right)$ es un equilibrio de Nash. Si no, supongamos que existe $x_{2}^{\prime} \neq x_{2}^{*}$ tal que

$$
f_{2}\left(x_{1}^{*}, x_{2}^{*}\right)<f_{2}\left(x_{1}^{*}, x_{2}^{\prime}\right),
$$

para algún $x_{2}^{\prime} \in S_{2}\left(x_{1}^{*}\right)$. Especificamente, supongamos

$$
f_{2}\left(x_{1}^{*}, x_{2}^{*}\right)+2 \epsilon<f_{2}\left(x_{1}^{*}, x_{2}^{\prime}\right)
$$


para algún $\epsilon>0$.

La continuidad de $\tilde{S}_{2}$ implica la existencia de una sucesión $\tilde{x}_{2}^{k} \rightarrow x_{2}^{*}$ tal que $\tilde{x}_{2}^{k} \in \tilde{S}_{2}\left(x_{1}^{k}\right)$ para toda $k \in \mathbb{N}$. Por tanto, la continuidad de $f_{2}$, implica la existencia de una $n$ suficientemente larga, tal que

$$
\left|f_{2}\left(x_{1}^{n}, \tilde{x}_{2}^{n}\right)-f_{2}\left(x_{1}^{*}, x_{2}^{\prime}\right)\right|<\epsilon
$$

$\mathrm{y}$

$$
\left|f_{2}\left(x_{1}^{n}, x_{2}^{n}\right)-f_{2}\left(x_{1}^{*}, x_{2}^{*}\right)\right|<\epsilon
$$

Por lo tanto,

$$
f_{2}\left(x_{1}^{n}, x_{2}^{n}\right)<f_{2}\left(x_{1}^{n}, \tilde{x}_{2}^{n}\right)
$$

y de esta forma se contradice la optimalidad $x_{2}^{n}$ en el $n$-ésimo paso del algoritmo I. De manera similar se cumple para la función de pago del jugador 1.

Ejemplo 3.11. Consideremos el caso de una cola en Tándem con dos servidores, donde cada servidor lo consideramos con una tasa de servicio exponencial, independientes e idénticamente distribuidos con tasas $\mu_{1}$ y $\mu_{2}$, respectivamente. Asumiremos también un límite superior el cual denotaremos como $u$, donde $u>\mu_{1} \vee \mu_{2}$. Hemos supuesto que para la entrada al servidor 1 , así como la capacidad entre el servidor 1 y 2 serán considerados con capacidad infinita. El rendimiento del sistema será $\frac{\mu_{1}}{\mu_{2}-\mu_{1}}$ si $\mu_{1}<\mu_{2}$ e igual a infinito en otro caso

Las funciones de pago de cada uno de los servidores, que podemos contemplar como los jugadores en el juego estarán definidas como sigue:

$$
f_{i}\left(\mu_{1}, \mu_{2}\right):=p_{i}\left(\mu_{1} \wedge \mu_{2}\right)-c_{i}\left(\mu_{i}\right)-g\left(\frac{\mu_{1}}{\mu_{2}-\mu_{1}}\right),
$$

donde $f_{i}$ corresponde a la función de costo que corresponde únicamente a la tasa de servicio, $p_{i}$ que será denominada como función de ganancia, $c_{i}$ la función de costo y por último $g$ que será la función inventario, con el siguiente espacio de estrategia:

$$
\begin{aligned}
& \tilde{S}_{1}\left(\mu_{2}\right)=\left\{\mu_{1}: 0 \leq \mu_{1} \leq \mu_{2}\right\} \\
& \tilde{S}_{2}\left(\mu_{1}\right)=\left\{\mu_{2}: \mu_{1} \leq \mu_{2} \leq u\right\}
\end{aligned}
$$

Observando que $g(\cdot)$ es una función creciente y cóncava, se cumple que $f_{1}\left(\mu_{1}, \mu_{2}\right)$ y $f_{2}\left(\mu_{1}, \mu_{2}\right)$ son funciones supermodulares. 
Además asumimos que todas las funciones de costo y beneficio son funciones continuas y $\lim _{x \rightarrow \infty} g(x)=\infty$ (lo que significa que que las tasas de servicio son muy cercanas entre sí).

$\tilde{S}_{1}$ y $\tilde{S}_{2}$ satisfacen la propiedad ascendente ya que si consideramos $\mu_{1}$ y $\mu_{1}^{\prime}$ donde $\mu_{1} \leq \mu_{1}^{\prime}$ y tenemos que $\widetilde{S}_{2}\left(\mu_{1}\right)=\left\{\mu_{2}: \mu_{1} \leq \mu_{2} \leq u\right\} \prec\left\{\mu_{2}: \mu_{1}^{\prime} \leq \mu_{2} \leq u\right\}=\tilde{S}_{2}\left(\mu_{1}^{\prime}\right)$, se cumple para cualquier elemento de los espacios de estrategia, y la continuidad la observamos suponiendo que $\mu_{2}^{k} \rightarrow \mu_{2}^{*}$, si tenemos que $\mu_{1}^{*}=0$ entonces tenemos que $\mu_{1}^{k} \equiv 0$, pero si tenemos que $\mu_{1}^{*}>0$, entonces hagamos $\mu_{1}^{k}=\mu_{2}^{k}-\mu_{2}^{*}+\mu_{1}^{*}$, por tanto, se cumplen las condiciones requeridas en el Teorema 3.10 y los perfiles de estrategia siguiendo el algoritmo I convergen a un equilibrio de Nash.

Si tomamos de manera adecuada las condiciones tenemos un caso particular

Tomemos todas las funciones lineales y para situaciones triviales, asumimos que

$$
p_{i}>c_{1}+c_{2}, \quad u>\frac{g}{p_{1}-c_{1}}
$$

La primera de las desigualdades nos garantiza que la unidad beneficio pueda exceder la unidad de costos de operación, sin la segunda desigualdad tendriamos que el límite superior $u$ sobre la tasa de servicio sería innecesario.

Consideremos en el ejemplo que

$$
\frac{\delta f_{1}}{\delta \mu_{1}}=0 \quad \mathrm{y} \quad \frac{\delta f_{2}}{\delta \mu_{2}}=0
$$

entonces las funciones de pago de ambos jugadores las tendremos de la siguiente manera, considerando $p_{i}\left(\mu_{i}\right)=p_{i} \mu_{i}$ y $c_{i}\left(\mu_{i}\right)=c_{i} \mu_{i}$ :

$$
\begin{aligned}
f_{i}\left(\mu_{1}, \mu_{2}\right) & :=p_{i}\left(\mu_{1} \wedge \mu_{2}\right)-c_{i}\left(\mu_{i}\right)-g\left(\frac{\mu_{1}}{\mu_{2}-\mu_{1}}\right) \\
& =p_{i}\left(\mu_{1}\right)-c_{i}\left(\mu_{i}\right)-g\left(\frac{\mu_{1}}{\mu_{2}-\mu_{1}}\right) \\
& =p_{i} \mu_{1}-c_{i} \mu_{i}-\frac{g \mu_{1}}{\mu_{2}-\mu_{1}}
\end{aligned}
$$

y derivando, obtenemos

$$
\mu_{1}=\mu_{2}-\left(\frac{g \mu_{2}}{p_{1}-c_{1}}\right)^{1 / 2} \quad \mathrm{y}
$$




$$
\mu_{2}=\mu_{1}+\left(\frac{g \mu_{1}}{c_{2}}\right)^{1 / 2}
$$

Hay que observar el caso $\mu_{1} \geq 0$ implica que ya sea $\mu_{1}=\mu_{2}=0$ o que $\mu_{2}>$ $g /\left(p_{1}-c_{1}\right)$ y obtenemos que tanto $\mu_{1}$ como $\mu_{2}$ son crecientes.

Por lo tanto, para todos los valores de parámetros, el punto

$$
\mu_{1}^{*}=\mu_{2}^{*}=0
$$

Cuando se tiene el caso en el que

$$
u \geq \frac{g\left(p_{1}-c_{1}\right)}{\left(p_{1}-c_{1}-c_{2}\right)^{2}}
$$

(Notar que $\left.\frac{g\left(p_{1}-c_{1}\right)}{\left(p_{1}-c_{1}-c_{2}\right)^{2}}>\frac{g}{\left(p_{1}-c_{1}\right)}\right)$ y tenemos que cuando $\mu_{2}=g\left(p_{1}-c_{1}\right) /\left(p_{1}-\right.$ $\left.c_{1}-c_{2}\right)^{2}$ y obtenemos $\mu_{1}=g c_{2} /\left(p_{1}-c_{1}-c_{2}\right)^{2}$, que serán otros equilibrios adicionales

$$
\mu_{1}^{*}=\frac{g c_{2}}{\left(p_{1}-c_{1}-c_{2}\right)^{2}}, \quad \mu_{2}^{*}=\frac{g\left(p_{1}-c_{1}\right)}{\left(p_{1}-c_{1}-c_{2}\right)^{2}}
$$

y el otro equilibrio lo obtenemos cuando $u=\mu_{2}$, entonces

$$
\mu_{1}^{*}=u-\left(\frac{g u}{p_{1}-c_{1}}\right)^{1 / 2}, \mu_{2}^{*}=u
$$

Obteniendo estos tres puntos de equilibrio se pueden analizar con la teoría clásica de colas usando 1.31 y 1.32 . 
El caso en el que $\mu_{1}^{*}=\mu_{2}^{*}=0$, lo omitiremos ya que podemos interpretar este caso que se tienen que cada uno de los servidores tiene una tasa de servicio 0 , que significa que no están atendiendo clientes y por tanto la cantidad de tiempo que pasará un cliente en el sistema tenderá a $\infty$.

Para el caso en el que el punto de equilibrio es $\mu_{1}^{*}=\frac{g c_{2}}{\left(p_{1}-c_{1}-c_{2}\right)^{2}}$ y $\mu_{2}^{*}=\frac{g\left(p_{1}-c_{1}\right)}{\left(p_{1}-c_{1}-c_{2}\right)^{2}}$ tenemos que haciendo el análisis con la teoría clásica de sistema de espera obtenemos el número promedio de clientes en el sistema y la cantidad promedio de tiempo que un cliente para en el sistema.

$$
\begin{aligned}
L & =\frac{\lambda\left(p_{1}-c_{1}-c_{2}\right)^{2}}{g c_{2}-\lambda\left(p_{1}-c_{1}-c_{2}\right)^{2}}+\frac{\lambda\left(p_{1}-c_{1}-c_{2}\right)^{2}}{g\left(p_{1}-c_{1}\right)-\lambda\left(p_{1}-c_{1}-c_{2}\right)^{2}} \\
W & =\frac{\left(p_{1}-c_{1}-c_{2}\right)^{2}}{g c_{2}-\lambda\left(p_{1}-c_{1}-c_{2}\right)^{2}}+\frac{\left(p_{1}-c_{1}-c_{2}\right)^{2}}{g\left(p_{1}-c_{1}\right)-\lambda\left(p_{1}-c_{1}-c_{2}\right)^{2}}
\end{aligned}
$$

Y por último, concluimos con el análisis del último punto de equilibrio, cuando tenemos que $\mu_{1}^{*}=u-\left(\frac{g u}{p_{1}-c_{1}}\right)^{1 / 2}$ y $\mu_{2}^{*}=u$

$$
\begin{gathered}
L=\frac{\lambda}{u-\left(\frac{g u}{p_{1}-c_{1}}\right)^{1 / 2}-\lambda}+\frac{\lambda}{u-\lambda}=\frac{\lambda\left[(u-\lambda)\left(p_{1}-c_{1}\right)+\left[(g u)\left(p_{1}-c_{1}\right)\right]^{\frac{1}{2}}\right]}{\left(p_{1}-c_{1}\right)(u-\lambda)-g u} \\
W=\frac{1}{u-\left(\frac{g u}{p_{1}-c_{1}}\right)^{1 / 2}-\lambda}+\frac{1}{u-\lambda}=\frac{(u-\lambda)\left(p_{1}-c_{1}\right)+\left[(g u)\left(p_{1}-c_{1}\right)\right]^{\frac{1}{2}}}{\left(p_{1}-c_{1}\right)(u-\lambda)-g u}
\end{gathered}
$$

Por lo tanto, siguiendo el algortimo de Round Robin, la convergencia a un equilibrio no sólo depende del rango del parámetro, sino también depende del perfil de estrategia inicial.

A continuación mostraremos las siguientes cuatro posibilidades: 
- Si $\mu_{2} \leq g /\left(p_{1}-c_{1}\right)$, entonces ambos servidores llegan a la solución del equilibrio 3.1. Lo que podemos traducir como, que la tasa de servicio del segundo servidor si comienza siendo menor o igual a tal restricción, entonces ambos servidores apagan su servicio inmediatamente

- Si $g /\left(p_{1}-c_{1}\right)<\mu_{2}^{0}<u \wedge g\left(p_{1}-c_{1}\right) /\left(p_{1}-c_{1}-c_{2}\right)^{2}$, entonces después del primer movimiento del servidor 1 (independiente de la dirección), el servidor 2 decrecerá su tasa. Ya que $\mu_{1}\left(\mu_{2}\right)$ domina $\mu_{2}\left(\mu_{1}\right)$ en este parámetro, hará que ambos servidores decrezcan sus tasas hasta llegar al equilibrio 3.1.

- Si $\mu_{2}^{0}=\mu_{2}^{*}=g\left(p_{1}-c_{1}\right) /\left(p_{1}-c_{1}-c_{2}\right)^{2} \leq u$, entonces el servidor 1 mueve a $\mu_{1}^{*}$ (en el primer paso) y el equilibrio 3.2 es alcanzado.

- Si $g\left(p_{1}-c_{1}\right) /\left(p_{1}-c_{1}-c_{2}\right)^{2} \leq \mu_{2}^{0} \leq u$, entonces, después del primer movimiento del servidor 1 (independiente de su dirección), el servidor 2 incrementará su tasa (ya que $\mu_{2}\left(\mu_{1}\right)$ domina $\mu_{1}\left(\mu_{2}\right)$ en este parámetro). Por tanto, ambos servidores incrementarán sus tasas hasta llegar al equilibrio 3.2.

\subsubsection{Algoritmo para $n$ jugadores}

Si consideramos $n$-jugadores, el algoritmo de Round Robin será como mostramos a continuación:

El perfil de estrategias denotaremos por $x:=\left(x_{i}\right)_{i=1}^{n}$ con $x_{i} \in S_{i}$. Denotaremos $x_{-i}$, como el vector $x$ sin el $i$-ésimo componente, de manera similar $x_{-(i, j)}$ indicará el vector $x$ sin el $i$-ésimo y sin el $j$-ésimo componentes.

Roun Robin para $n$ jugadores

En el paso $k$ el jugador 1 primero encuentra la estrategia $x_{1}^{k}$ que maximiza su función de utilidad $f_{1}$, dadas las estrategias de los otros jugadores en el paso previo

$$
x_{1}^{k}=\underset{x_{1} \in S_{1}}{\operatorname{argmax}} f_{1}\left(x_{1}, x_{-1}\right), \text { donde } x_{-1}=x_{-1}^{k-1}
$$

después el jugador 2, encuentra la estrategia $x_{2}^{k}$ que maximiza su función de utilidad $f_{2}$, dadas las más recientes estrategias de los otros jugadores, incluyendo la nueva estrategia del jugador 1 , de este modo

$$
x_{2}^{k}=\underset{x_{2} \in S_{2}}{\operatorname{argmax}} f_{2}\left(x_{2}, x_{-2}\right), \text { donde } x_{-2}=\left(x_{1}^{k} x_{-(1,2)}^{k-1}\right)
$$

De igual forma para el jugador 3 , este encuentra la estrategia $x_{3}^{k}$ que maximiza su función de utilidad $f_{3}$ : 


$$
x_{3}^{k}=\underset{x_{3} \in S_{3}}{\operatorname{argmax}} f_{3}\left(x_{3}, x_{-3}\right), \text { donde } x_{-3}=\left(x_{1}^{k}, x_{2}^{k} x_{-(1,2,3)}^{k-1}\right)
$$

$\mathrm{Y}$ esto se repite en los siguientes pasos de manera sucesiva.

\section{3. $\quad S$-modularidad}

En esta sección describiremos el concepto de $S$-modularidad la cual será vista como una generalización de la supermodularidad y submodularidad, que permite el tratamiento de la supermodularidad y submodularidad simultáneamente.

Definición 3.12. Denotaremos $(i, j) \in \tilde{S}_{+}$si las funciones de pago $f_{i}$ y $f_{j}$ son supermodulares en $\left(x_{i}, x_{j}\right)$.

Denotaremos $(i, j) \in \tilde{S}_{-}$si las funciones de pago $f_{i}$ y $f_{j}$ son submodulares en $\left(x_{i}, x_{j}\right)$.

Observación 3.13. Para trabajar la $S$-modularidad, necesitaremos una condición clave, la cual restringe las posibles relaciones $\tilde{S}_{+}$y $\tilde{S}_{-}$entre todos los jugadores.

Definición 3.14. Condición de $S$-modularidad

Si $(i, j) \in \tilde{S}_{+}$entonces para cualquier $h \neq i, j$, ya sea $(h, i),(h, j) \in \tilde{S}_{+}$o $(h, i),(h, j) \in$ $\tilde{S}_{-}$.

Si $(i, j) \in \tilde{S}_{-}$entonces para cualquier $h \neq i, j$, ya sea $(h, i) \in \tilde{S}_{+},(h, j) \in S_{-}$o $(h, i) \in$ $\tilde{S}_{-},(h, j) \in \tilde{S}_{+}$.

Observación 3.15. La condición de la $S$-modularidad reduce el número total de las relaciones entre todas las parejas de jugadores de $\left(\begin{array}{l}n \\ 2\end{array}\right)$ a $n-1$.

Para ver esto empecemos tomando al jugador 1 , una vez que $(1, i) \in \tilde{S}_{+}$o $\tilde{S}_{-}$es determinado para todos los $i$ jugadores, donde $i=1, \ldots, n$, entonces las relaciones para las demás parejas quedan también fijas.

Ejemplo 3.16. Consideremos al jugador 2 , si $(1,2) \in \tilde{S}_{+}$entonces $(2, h)$ debe tener la misma relación la de la pareja $(1, h), \forall h$, con $h \neq 1,2$, si $(1,2) \in \tilde{S}_{-}$, entonces la relación de $(2, h)$ debe ser opuesta que la de $(1, h)$. De manera similar, la relación entre las parejas $(2,3)$ determinará la relación entre $(3, h) \forall h=4, \ldots n$, se concluye que es solamente analizar las relaciones de las $n-1$ parejas consecutivas.

Teorema 3.17. Consideremos un juego de $n$ jugadores. Supongamos que el algoritmo Round Robin es seguido y la condición de $S$-modularidad está en vigor, además de que todas las funciones de pago son continuas y los espacios de estrategia para cada 
jugador $i, \tilde{S}_{i}\left(x_{-i}\right)$ es un sublátiz compacto para cada $x_{-i}$ y es continua en $x_{-i}$, entonces tenemos que los perfiles de estrategia convergen a un equilibrio de Nash siempre que lo siguiente se satisfaga:

- si $(i, j) \in \tilde{S}_{+}$, entonces $\tilde{S}_{i}\left(x_{-i}\right)$ es ascendete en $x_{j}$ y $\tilde{S}_{j}\left(x_{-j}\right)$ es ascendente en $x_{i}$.

- si $(i, j) \in \tilde{S}_{-}$, entonces $\tilde{S}_{i}\left(x_{-i}\right)$ es descendente en $x_{j}$ y $\tilde{S}_{j}\left(x_{-j}\right)$ es descendente en $x_{i}$.

- El perfil de estrategia es tal que cada juador en un grupo empieza en el punto más pequeño de su espacio de estrategia, mientras que cada jugador en el otro grupo empieza en el punto más grande de su espacio de estrategia.

\section{DEMOSTRACIÓN}

Supongamos que el jugador 1 empieza en el punto más pequeño de su espacio de estrategia. Entonces debemos tener $x_{1}^{1}>x_{1}^{0}$. Además, que para cada jugador $i$ en el mismo grupo también se cumple $x_{i}^{1}>x_{i}^{0}$, ya que $x_{i}^{0}$ es el punto más pequeño del espacio de estrategia del jugador $i$. Ahora, para cada jugador $j$ en el otro grupo, tenemos que $x_{j}^{1}<x_{j}^{0}$ ya que es el punto más grande del espacio de estrategia del jugador $j$.

En el siguiente paso, para cada jugador $i$ en el mismo grupo que el jugador 1, debemos tener que $x_{i}^{2}>x_{i}^{1}$. Este incremento no sólo es mejorado por el incremento en la estrategia de todos los otros jugadores en el mismo grupo, sino también por el decredimiento en estrategia de los jugadores en el otro grupo. De manera similar se cumple que para cada $j$ en el otro grupo, se tiene que $x_{j}^{2}<x_{j}^{1}$

Este paso es repetido para todos los pasos subsecuentes y como resultado obtenemos que para jugador $h$, la sucesión de estrategias $x_{h}^{k}$, con $k=1,2, \ldots$, es o una sucesión creciente o una sucesión decreciente. La convergencia se cumple de la compacidad y la monotonicidad de los espacios de estrategia y el equilibrio de Nash viene de la continuidad de las funciones de pago, así como de la continuidad de los espacios de estartegia. De igual manera que cuando se tienen 2 jugadores.

Observación 3.18. La condición sobre el perfil de estrategia inicial puede ser reemplazada por la siguiente condición ligeramente débil: Suponiendo que el jugador 1 está en el grupo 1, las estrategias de los demás jugadores en el mismo grupo, con la posible excepción del jugador 1, se mueven en la misma dirección, mientras que los del otro grupo se moverán en dirección opuesta. 
Ejemplo 3.19. ( $n$ colas en tándem)

Recordando el Ejemplo 3.11, es fácil poder extender este ejemplo a $n$ Colas en Tándem, suponiendo que el servidor 1 toma trabajo de una fuente infinita, cada trabajo va a través de los $n$ servidores secuencialmente y abandona la cola en el servidor $n$. También suponemos que cada bucle entre los servidores tiene capacidad infinita.

Cada servidor $i$ corresponde a un jugador, con tasa de servicio $\mu_{i}$, que corresponde a la estrategia del jugador $i$.

Consideremos el espacio de estrategia de la siguiente manera:

$$
\tilde{S}_{1}=\left\{\mu_{1}: 0 \leq \mu_{1} \leq \mu_{i}\right\} \quad \text { y } \quad \tilde{S}_{i}=\left\{\mu_{i}: \mu_{1} \leq \mu_{i} \leq u\right\}
$$

observando que los espacios de estrategia $\tilde{S}_{1}$ y $\tilde{S}_{i}$ con $i=2, \ldots, n$ cumplen la propiedad ascendente, así como la continuidad.

Asumiremos que $g_{i}(\cdot)$ es creciente y $\lim _{x \rightarrow \infty} g_{i}(x)=\infty \forall i=1, \ldots, n$. Además para $i=2$, necesitaremos la convexidad de $g_{2}(\cdot)$, así como la supermodularidad de $-g_{2}\left(\mu_{1} /\left(\mu_{2}-\mu_{1}\right)\right)$.

Así de esta manera, siguiendo el algoritmo Round Robin nos dirigimos a un equilibrio de Nash. La única diferencia para el caso de 2 servidores 3.11, hay un requerimiento adicional sobre el perfil de estrategia inicial (ya que todos los jugadores pertenecen a un grupo, ellos deberán empezar en el punto más pequeño $\left(\mu_{i}=0\right)$ o el punto más grande $\left.\left(\mu_{i}=u\right)\right)$.

\subsection{Optimizadores ordenados}

En esta sección se abordará el caso en el que nos encontremos con múltiples optimizadores (o maximizadores) cuando en cada paso del algoritmo Round Robin, una función de pago es maximizada, además permitimos vectores de estrategia.

Notación 3.20. $M(y):=\operatorname{argmax}_{x \in \tilde{S}_{1}} f(x, y)$

Teorema 3.21. Supongamos dos espacios de estrategia $\tilde{S}_{1}$ y $\tilde{S}_{2}$, tal que $x \in \tilde{S}_{1}$ y $y \in \tilde{S}_{2}$, además de que $\widetilde{S}_{1}, \tilde{S}_{2}$ son sublátices, también supongamos que $f(x, y)$ es una función supermodular en $x$ y supermodular en $(x, y)$. Para $y \in \widetilde{S}_{2}$, entonces, $M(y)$ es ascendente en y $\left(y \leq y^{\prime} \Rightarrow M(y) \prec M\left(y^{\prime}\right)\right)$.

Observación 3.22. Para cualquier $y \in \tilde{S}_{2}$ se cumple que $M(y)$ es una látiz, ya que por la propiedad ascendente de $M(y)$ cuando $y \leq y^{\prime}$ se cumple que $x \in M(y)$ y $x^{\prime} \in M\left(y^{\prime}\right)$, entonces $x \wedge x^{\prime} \in M(y)$ y $x \vee x^{\prime} \in M\left(y^{\prime}\right)$ y si permitimos que $y=y^{\prime}$, tenemos que implica que $M(y)$ es un látiz. 
Teorema 3.23. Supongamos que $f(x, y)$ es supermodular en $x$ y submodular en $(x, y)$, entonces $M(y)$ es descendente en $y$.

\section{DemostracióN}

Supongamos $y \leq y^{\prime}, x \in M(y)$, tenemos

$$
\begin{array}{cc} 
& f\left(x \vee x^{\prime}, y\right)-f(x, y) \\
\geq & f\left(x \vee x^{\prime}, y^{\prime}\right)-f\left(x, y^{\prime}\right) \\
\geq & f\left(x^{\prime}, y^{\prime}\right)-f\left(x \wedge x^{\prime}, y^{\prime}\right) \\
\geq & 0
\end{array}
$$

donde la primer desigualdad se cumple por la suposición de la submodularidad en $(x, y)$ y por las diferencias crecientes, la segunda desigualdad se tiene por el hecho de que $f(x, y)$ es supermodular en $x 2.47$ y la tercer desigualdad de que $x^{\prime} \in M\left(y^{\prime}\right)$. Por tanto,

$$
f\left(x \vee x^{\prime}, y\right) \geq f(x, y)
$$

ya que $x \in M(y)$, tenemos que $x \vee x^{\prime} \in M(y)$, entonces

$$
0=f\left(x \vee x^{\prime}, y\right)-f(x, y)
$$

De 3.4, se cumple que todas las desigualdades deben ser igualdades, en particular

$$
f\left(x \wedge x^{\prime}, y^{\prime}\right)=f\left(x^{\prime}, y^{\prime}\right)
$$

Esto es, $x \wedge x^{\prime} \in M\left(y^{\prime}\right)$, ya que $x \in M\left(y^{\prime}\right)$.

Nota 3.24. De igual manera se cumple que $M(y)$ es látiz.

Observación 3.25. Los jugadores en el grupo correspondiente a $x$, todos deberían pertenecer a $\tilde{S}_{+}$, si cada uno de ellos, cuando se empareja con cualquier jugador en el grupo corresponde a $y$, pertenece a $\tilde{S}_{-}$. Cuando múltiples optimizadores son presentados, necesitamos ser más específicos sobre el algoritmo Round Robin, en particular, que solución elegir en cada paso y pasar al siguiente paso.

Definición 3.26. $M_{i}^{k}$, denotará el conjunto de optimizadores del jugador $i$ en el paso $k$. 
Definición 3.27. Definimos $z^{k}$, como el elemento más pequeño de $M_{i}^{k}$ y $\overline{z^{k}}$, como el elemento más grande de $M_{i}^{k}$.

Debemos considerar varios casos:

- En el primer caso consideramos que ambas funciones son supermodulares en $x$ y $y$, además, ser supermodular en $(x, y)$. Supongamos como principio, empezar en $\left(x_{0}, y_{0}\right)$, con $x_{0}$ y $y_{0}$, siendo los elementos más pequeños de $S_{1}$ y $S_{2}$, respectivamente. Supongamos que elegimos $\underline{x^{1}}$ como la solución para pasar al jugador 2, después el jugador elige $\underline{y^{1}}$, como la solución. Entonces, en el paso 2 se comienza con el elemento más pequeño, $\left(\underline{x^{1}}, \underline{y^{1}}\right)$ de $M_{1}^{1} \times M_{2}^{1}$ y continuamos eligiendo los elementos más pequeños en cada paso. Nos damos cuenta que de esta manera, el algoritmo produce una sucesión de perfiles monótonamente crecientes, $\left\{\left(\underline{x^{k}}, \underline{y^{k}}\right)\right\}$, el cual convergerá al límite $\left(\underline{x^{*}}, \underline{y^{*}}\right)$ y el límite es el equilibrio de Nash más pequeño, en el sentido que será dominado por cualquier otro equilibrio de Nash.

- Como segundo caso e igualmente podemos considerar, podemos elegir el elemento más grande del espacio de perfil conjunto $S$, como la solución inicial y elegir el elemento más grande de los sublátices de maximizadores en cada paso. De esta manera, el algortimo produce una sucesión de perfiles monótonamente decrecientes, $\left\{\left(\overline{x^{k}}, \overline{y^{k}}\right)\right\}$, el cual convergerá al límite $\left(\overline{x^{*}}, \overline{y^{*}}\right)$, el equilibrio de Nash, más grande.

- Cuando las funciones de pago son submodulares en $(x, y)$ y supermodulares en $x$ y $y$, los resultados de los incisos anteriores se mantienen a excepción de algunas condiciones, que mostraremos a continuación:

a) El jugador 1 comienza en el elemento más pequeño, mientras que el jugador 2 comienza en el elemento más grande de sus respectivos espacios de estrategia.

b) El jugador 1 elige el elemento más pequeño del sublátiz de maximizadores en cada paso y el jugador 2 elige el elemento más grande en cada paso.

De esta manera, el algoritmo produce una sucesión creciente $\left\{\underline{x^{k}}\right\}$ y una sucesión decreciente $\left\{\overline{y^{k}}\right\}$, que convergen a $\underline{x^{k}}$ y a $\overline{y^{k}}$, respectivamente y $\left(\underline{x^{*}}, \overline{y^{*}}\right)$ es un equilibrio de Nash.

- Para el caso con $n$ jugadores, agruparemos a los $n$ jugadores en 2 grupos: $\left(i_{1}, \ldots, i_{k}\right)$ y $\left(j_{1}, \ldots, j_{l}\right)$ con $k+l=n$, Por tanto, 


$$
x:=\left(x_{i_{1}}, \ldots, x_{i_{k}}\right), \quad y:=\left(\left(y_{j_{1}}, \ldots, y_{j_{l}}\right)\right)
$$

nos coloca en el Teorema 3.23.

Definición 3.28. Diremos que los jugadores que corresponden a las componentes en y, como $x$-jugadores ( $y$-jugadores). Siguiendo lo anterior y analizando el caso para 2-jugadores, empezamos con las soluciones iniciales más pequeñas (más grandes) para los $x$-jugadores y las soluciones iniciales más grandes (más pequeñas) para los $y$-jugadores y en cada paso siempre elegir los maximizadores más grandes (más pequeños), $\overline{y^{k}}\left(\underline{y^{k}}\right)$, para los $y$-jugadores.

Teorema 3.29. Consideremos el juego de $n$ jugadores del Teorema 3.17, permitiendo que las estrategias de los $i$ jugadores sean vectores. Además de las condiciones en el Teorema 3.17, asumimos que la función de pago $f_{i}\left(x_{1} \ldots, x_{n}\right), \forall i=1, \ldots, n$, es supermodular en $x_{j} \forall j=1, \ldots, n$, entonces, en cada paso $k$, el conjunto de maximizadores de $f_{i}, M_{i}^{k}$, es un látiz, $\forall i=1, \ldots, n$. Además, el algoritmo converge a un equilibrio de Nash $\left(\underline{x}^{*}, \bar{y}^{*}\right)\left(\right.$ o $\left.\left(\bar{x}^{*}, y^{*}\right)\right)$; y cualquier equilibrio de Nash $\left(x^{\prime}, y^{\prime}\right)$ satisface: $x^{\prime} \geq \underline{x}^{*}, y^{\prime} \leq \bar{y}^{*}\left(x^{\prime} \leq \bar{x}^{*}, y^{\prime} \geq \underline{y}^{*}\right)$

\section{Demostración}

Este Teorema lo demostraremos por inducción. Observemos que se cumple que $x^{\prime} \geq x^{0}$ y $y^{\prime} \leq y^{0}$.

Supongamos que para algún paso $k$, tenemos lo siguiente

$$
x^{\prime} \geq x^{k} \text { y } y^{\prime} \leq y^{k}
$$

Supongamos el paso $k+1$, empezamos analizando con el movimiento del jugador 1. Dado 3.5, si el jugador es un $x$-jugador, su estrategia actualizada será dominada por la estrategia correspondiente en $x^{\prime}$, la cual permanecerá siendo la misma (ya que es un equilibrio de Nash). Si el jugador es un $y$-jugador, su estrategia actualizada dominará la correspondiente estrategia en $y^{\prime}$, la cual también permanece sin cambios. Por tanto,

$$
x_{1}^{\prime} \geq x_{1}^{k} \text { y } y_{1}^{\prime} \leq y_{1}^{k}
$$

Este argumento entonces continua a la actualización de los otros jugadores y además para los siguientes pasos. Entonces por hipótesis de inducción en $3.5 \forall k$ y tomando límite sobre $k$ y produce la relación deseada, que es 


$$
x^{\prime} \geq \underline{x}^{*} \text { y } y^{\prime} \leq \bar{y}^{*}
$$

\subsection{Bifurcación}

Para abordar el caso de una bifurcación, optaremos por considerar lo siguiente, anteriormente vimos que las condiciones requeridas en el Teorema 3.17 limitan la relación entre los $n$ jugadores y los analiza únicamente como dos grupos, donde únicamente se encuentra $\tilde{S}_{+}$en un grupo y $\tilde{S}_{-}$, en este capítulo se aborda un caso en particular tomando un nuevo algoritmo, que nombraremos como algoritmo II que nos hará encontrar un caso particular.

A continuación abordaremos un caso que infringe las condiciones en el Teorema 3.17:

Supongamos que $(i, j) \in \tilde{S}_{-}$para $i, j=1, \ldots, n-1$, mientras que $(i, n) \in \tilde{S}_{+}$para $i=1, \ldots, n-1$. Además supondremos que las estrategias de los jugadores son escalares y el espacio de estrategia es un sublátiz compacto que es independiente de cualquier otra estrategia de los jugadores.

Definición 3.30. Denotemos por $x:=\left(x_{i}\right)_{i=1}^{n-1}$, el conjunto de estrategias de los primeros $n-1$ jugadores y $y:=x_{n}$ la estrategia del último jugador (donde el último jugador será $n$ ).

Definición 3.31. De manera similar, denotaremos con $\tilde{S}_{x}$, el espacio de estrategia conjunta de los primeros $n-1$ jugadores y con $\tilde{S}_{y}$ nos referiremos al espacio de estrategia del jugador $n$.

\section{Algoritmo II}

a) Se empezará el algoritmo con $\left(x^{0}, y^{0}\right)$, donde $x^{0}$ será el elemento más pequeño de $\tilde{S}_{x}$ y $y^{0}$ el elemento más grande de $\tilde{S}_{y}$.

b) En cada paso $k \geq 1$, cada una de las funciones de pago será maximizada con las estrategias de los otros jugadores del paso $k-1$, además, en cada paso siempre elegiremos el maximizador más pequeño $x^{k}$ y el maximizador más grande $y^{k}$ 
Observación 3.32. Debemos notar que el algoritmo $I$ usa un procedimiento de actualización secuencial entre los $n$ jugadores en cada paso de este, mientras que en el algoritmo $I I$ la actualización en cada paso es llevada a cabo simultaneamente entre todos los jugadores.

En el paso 1, se cumple lo siguiente:

$$
x^{1} \geq x^{0} \quad \text { y } \quad y^{1} \leq y^{0}
$$

lo anterior debido a que $x^{0}$ y $y^{0}$ son, respectivamente, los elementos más pequeño y más grande de $\widetilde{S}_{x}$ y $\widetilde{S}_{y}$.

En el paso 2, tenemos

$$
x^{0} \leq x^{2} \leq x^{1} \quad \text { y } \quad y^{1} \geq y^{2} \geq y^{0}
$$

la primera desigualdad de la ecuación anterior se cumple debido a la suposición hecha, ya que $x^{0}$ y $y^{0}$ son los elementos más pequeño y más grande de sus espacios de estrategia, respectivamente, la segunda desigualdad se cumple, debido a las supuestas relaciones de supermodularidad y submodularidad.

Por ejemplo, si consideramos al jugador $i \neq n$. El maximizador $x_{i}^{2}$ (de $f_{i}$ ) esta basado en $\left(x_{-i}^{1}, y^{1}\right)$, donde el maximizador $x_{i}^{1}$ (también de $f_{i}$ ) está basado en $\left(x_{-i}^{0}, y^{0}\right)$, ya que $(i, j) \in S_{-}$, para $j \neq n, i$, y $(i, n) \in S_{+}$, tenemos $x_{i}^{2} \leq x_{i}^{1}$. Siguiendo el mismo argumento, $y^{2} \geq y^{1}$, ya que los dos maximizadores son basados en $x^{1}$ y $x^{0}$, respectivamente.

En el paso 3, tenemos

$$
x^{2} \leq x^{3} \leq x^{1} \quad \text { y } \quad y^{2} \geq y^{3} \geq y^{1}
$$

Para argumentar la primera desigualdad, de nuevo consideremos al jugador $i \neq n$. Notar que $x_{i}^{3}$ es el maximizador de $f_{i}$ dado $\left(x_{-i}^{2}, y^{2}\right)$, mientras $x_{i}^{2}$ es el maximizador del mismo $f_{i}$, dado $\left(x_{-i}^{1}, y^{1}\right)$. Y $x^{3} \geq x^{2}$ se sigue de las relaciones de supermodularidad y submodularidad especificas.

Inductivamente, concluimos lo siguiente

$$
x^{2 k-2} \leq x^{2 k} \leq x^{2 k-1}, \quad x^{2 k} \leq x^{2 k+1} \leq x^{2 k-1}
$$

$\mathrm{y}$

$$
y^{2 k-2} \geq y^{2 k} \geq y^{2 k-1}, \quad y^{2 k} \geq y^{2 k+1} \geq y^{2 k-1}
$$

Por tanto, para $x$, tenemos dos sucesiones monótonas: $\left\{x^{2 k}\right\}$ que decrece a un límite $x^{L}$ y $\left\{x^{2 k-1}\right\}$ que crece a un límite $x^{U}$. De manera similar, $\left\{y^{2 k}\right\}$ crece a un 
límite $y^{U}$ y $\left\{y^{2 k-1}\right\}$ que decrece a un límite $y^{L}$, donde $x^{L} \leq x^{U}$ y $y^{L} \leq y^{U}$. En el peor de los casos, es posible que las dos sucesiones se mantengan constantes en la solución inicial y el algoritmo simplemente oscila entre los dos. Sin embargo, cuando $x^{L}=x^{U}=x^{*}$ y $y^{L}=y^{U}=y^{*}$, tenemos convergencia a un equilibrio de Nash $\left(x^{*}, y^{*}\right)$

Teorema 3.33. Consideremos un juego de $n$ jugadores como en el Teorema 3.17. Supongamos $(i, j) \in \tilde{S}_{-}$para $i, j=1, \ldots, n-1, \quad i \neq j \mathrm{y}(i, n) \in \tilde{S}_{+}$para $i=1, \ldots, n-$ 1. Siguiendo el algoritmo II, los perfiles de estrategia convergen a dos límites, donde uno estará dominando al otro.

Cuando los dos límites coinciden, el resultado es un equilibrio de Nash.

Nota 3.34. Lo anterior podemos considerarlo como un teorema que define ciertas condiciones para unicidad de puntos de equilibrio.

\subsection{Conclusiones}

En este capítulo desarrollamos los juegos $S$-modulares, que son un tipo de juegos más generales que los juegos supermodulares descritos en un capítulo anterior, ya que éstos nos permiten trabajar de manera similar ya sea con funciones supermodulares o funciones submodulares. A partir de ésto se enunciaron dos algoritmos:

- Algoritmo I, que es el algoritmo de Round Robin pero para juegos $S$-modulares y se presentaron ejemplos en Sistemas de Espera en Tándem, sin salidas entre servidores, ilustrando todos los conceptos enunciados con anterioridad.

- Algoritmo II, que es una adaptación del algoritmo I, la única variante que se presenta es que cada uno de los jugadores actualiza su estrategia dada la estrategia de cada uno de los jugadores en un paso anterior. Este algoritmo nos presenta una bifurcación y a partir de esto enunciamos el Teorema 3.33 que nos garantiza la unicidad de equilibrios de Nash en juegos $S$-modulares. 


\section{Capítulo 4}

\section{Conclusiones y Perspectivas}

\subsection{Conclusiones}

Primero en esta tesis desarrollamos los sistemas de espera $M / M / 1$, seguido del Teorema de Burke que nos dice que la distribución del tiempo entre salidas consecutivas del sistema $M / M / 1$ es idéntica a la distribución del tiempo entre llegadas. En segundo término se prosiguió con el ánalisis de la distribución de las salidas, la cual resulta ser la misma que la distribución de las llegadas y no se afecta por el mecanismo de servicio (exponencial) y con todo ésto analizamos un Sistema de Espera en Tándem.

En el capítulo 2 describimos las propiedades de los juegos supermodulares a partir de la definición de látices y sublátices, desarrollando propiedades topológicas en estos juegos y describiendo lo que son las funciones supermodulares acompañadas del conjunto de soluciones óptimas para los juegos supermodulares y las condiciones necesarias para la existencia de puntos de equilibrio en éstos y se concluye este capítulo con un algortimo que nos garantiza la existencia de dichos puntos, conocido como el algoritmo de Round Robin.

En el capítulo 3, desarrollamos los juegos $S$-modulares, que son un tipo de juegos más generales que los juegos supermodulares descritos en el capítulo anterior, ya que éstos nos permiten trabajar de manera similar, ya sea con funciones supermodulares o submodulares; a partir de esto se enunciaron dos algoritmos:

- Algoritmo I, que es el algoritmo de Round Robin, pero para juegos $S$-modulares y se presentaron ejemplos en sistemas de espera en tándem sin salidas entre 
servidores, ilustrando todos los conceptos enunciados con anterioridad.

- Algoritmo II, que es una adaptación del algoritmo I, la única variante que se presenta es que cada uno de los jugadores actualiza su estrategia dada la estrategia de cada uno de los jugadores en un paso anterior, este algoritmo nos presenta una bifurcación y a partir de esto enunciamos un teorema que nos garantiza la unicidad de equilibrios de Nash en juegos $S$-modulares.

Hay que destacar que los juegos s-modulares son apropiados para estudiar modelos en economía, sistemas de espera, entre otros, en los cuales el planteamiento de teoremas de punto fijo para la existencia de equilibrios de Nash es sumamente complicado y en contraparte el tratamiento de orden de los sistemas $s$-modulares facilita la existencia de equilibrios de Nash, a través de los algoritmos antes mencionados.

\subsection{Perspectivas}

A futuro se pretende:

- Aplicar la teoría desarrollada en sistemas que no sean de tipo tándem o que contengan salidas entre servidores.

- Obtener reglas de paro para los algoritmos $I$ y II desarrollados en esta tesis. 


\section{Bibliografía}

[1] Altman, E., Applications of dynamic games in queues. A.S. Nowak, K. Szajowski, eds. Advances in Dinamic Games: Applications to Economics, Finance, Optimization and Stochastic Control, Birkhäuser, Boston, (2005), 309-342.

[2] Altman, E. And Altman, Z., S-modular games and power control in wireless networks, IEEE Journal on Selected Areas in Communications, Vol. 48, No. 5 (2003), 839-842.

[3] E. Altman, T. Boulogne, R. El-Azouzi, T. Jiménez, And L. Wynter, A survey on networking games in telecommunications, Computers \& Operations Research, Vol. 33, (2006) 286-311.

[4] Amir, R., Supermodularity and complementarity in economics: An elementary survey, Southern Economic Journal, (2005), 636-660.

[5] Bhat,N., An Introduction to Queueing Theory, Birkhäuser, Boston, (2008).

[6] Burke, P. J., The output of a queuing system, Operations Research, Vol. 4, No. 6 (1956), 699-704.

[7] Chengnian, Non-Cooperative Power Control for Wireless Ad Hoc Networks with Repeated Games, IEEE Journal on Selected Areas in Communications, Vol. 25, No. 6, (2007), 1101-1112.

[8] Handouf, S. And SABIR, E., Strategic availability and cost-effective UAVbased flying access networks: S-modular game analysis, Nest Research Group, (2019), 1-12.

[9] Chee-Hock, NG. And Boon-Hee, S., Queueing Modelling Fundamentals, JOHN WILEY \& SONS, (1997). 
[10] Hsu, H. P., Theory and Problems of Probability, Random Variables and Random Processes, McGraw-Hill, first edition, (1997).

[11] Kakutani, S., A generalization of Brower's fixed point theorem., Duke Mathematical Journal, Vol. 8, (1941), 457-459.

[12] GLicksberg, L., A further generalization of the Kakutani fixed point theorem, with application to Nash equilibrium points, Proceedings of the American Mathematical Society, Vol. 3, (1952),170-174.

[13] Li, S. And Basar, T., Distributed algorithms for the computation of noncooperative equilibria, Automatica, Vol. 23, (1987), 523-533.

[14] Nash, J., Equilibrium points in n-person games, Proceedings of the National Academy of Sciences of the United States of America, Vol. 36, (1950), 48-49.

[15] Nash, J., Non-cooperative games, Annals of Mathematics, Second Series, Vol. 54, No. 2 (1951), 286-295.

[16] Pucci, R., A MAC Level strategy for dynamic resource allocation in cognitive radio tactical networks, IEEE ICC 2015 - Workshop on cognitive radios and networks for spectrum coexistence of satellite and terrestrial systems, (2015), 1658-1662.

[17] Rosen, J. B., Existence and uniqueness of equilibrium points for concave $\mathrm{N}$ person games, Econometrica, Vol. 33, (1965), 520-534

[18] Ross, S. M., Introduction to Probability Models, Academic Press, tenth edition, (2010).

[19] Deng, R., Yang, Z., Chen, J., Rahbari, N. and Chow, M., residential energy consumption scheduling: A coupled-constraint game approach, IEEE Transactions on Smart Grid, Vol. 5, no. 3, (2004), 1340-1350.

[20] Sundaram, R. K., A First Course in Optimization Theory, New York: Cambridge University Press, (1996).

[21] Topkis, D. M., Supermodularity and Complementarity, New Jersey: Princeton University Press, first edition, 1998.

[22] YAO, D., S-modular games, with queueing applications, Queueing Systems, Vol. 21, No. 3-4 (1995), 449-475. 
[23] Yu, G., QImeI, C., QIAng, Y. and Weinua, Z., Game-theoretic optimization for machine-type communications under QoS guarantee,IEEE Internet of Things Journal Vol. 6, Issue: 1, (2019), 719-800.

[24] Zapata, P., Economía Política y Otros Juegos. Una Introducción a los Juegos No Cooperativos. Facultad de Ciencias, UNAM, primera edición, (2007). 
Teoria de Juegos S-modulares con Aplicaciones a Sistemas de Espera en Tándem
En la Ciudad de México, se presentaron a las 17:00 horas del día 11 del mes de noviembre del año 2019 en la Unidad Iztapalapa de la Universidad Autónoma Metropolitana, los suscritos miembros del jurado:

DR. JULIO CESAR GARCIA CORTE

DR. VICTOR HUGO VAZQUEZ GUEVARA

DR. JOSE RAUL MONTES DE OCA MACHORRO
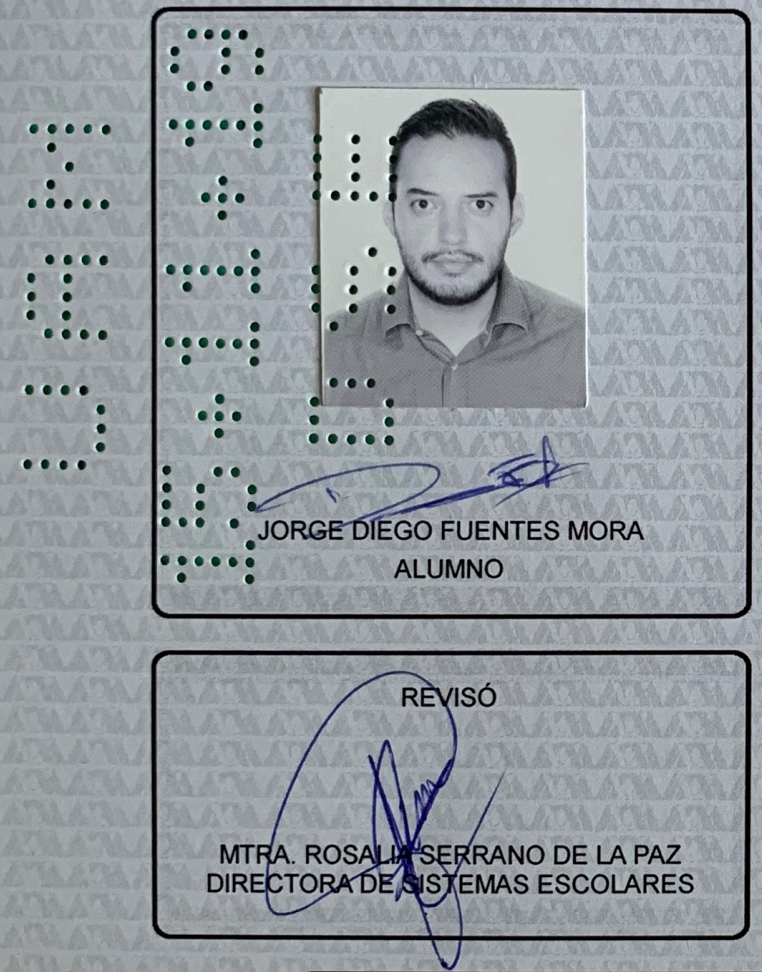

DIRECTOR DE LA DIVISIÓN DE CBI

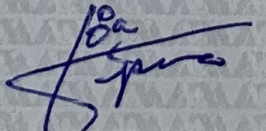

DR. JESUS ALBERTO OCHOA TAPIA

VOCAL

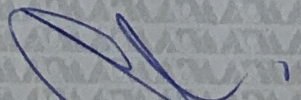

DR. VICTOR HUGO VAZQUEZZ GUEVARA
Bajo la Presidencia del primero y con carácter de Secretario el último, se reunieron para proceder al Examen de Grado cuya denominación aparece al margen, para la obtención del grado de:

MAESTRO EN CIENCIAS (MATEMÁTICAS)

DE: JORGE DIEGO FUENTES MORA

$y$ de acuerdo con el artículo 78 fracción III del Reglamento de Estudios Superiores de la Universidad Autónoma Metropolitana, los miembros del jurado resolvieron:

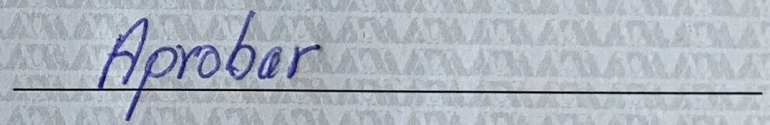

Acto continuo, el presidente del jurado comunicó al interesado el resultado de la evaluación $y$, en caso aprobatorio, le fue tomada la protesta. 
